\title{
Estimating the Unsteady Angle of Attack from Blade Pressure Measurements on the NREL Phase VI Rotor in Yaw using a Free-Wake Vortex Model
}

\author{
Tonio Sant ${ }^{*}$ \\ Delft University of Technology, Delft, The Netherlands, 2629HS and University of Malta, Msida, Malta, MSD 07 \\ and \\ Gijs van Kuik $^{\dagger}$ and Gerard van Bussel ${ }^{*}$ \\ Delft University of Technology, Delft, The Netherlands, 2629HS
}

The unsteady aerodynamic phenomena associated with yawed wind turbines are still poorly understood and are therefore challenging to predict accurately. The main issues concern the geometry of the skewed vortical wake formed behind the turbine, the unsteady flow field at the rotorplane induced by the vortical wake, as well as the aerodynamic effects of unsteady flow over the blade sections. Blade pressure measurements on a rotating blade of a yawed wind turbine can be very useful in obtaining further insight of yaw aerodynamics. However, in doing so, knowledge of the time-dependent angle of attack and induced velocity distributions at the rotoplane is an indispensable requirement. This paper presents a method to derive such distributions for the NREL phase VI turbine using a free-wake vortex model. The study considered different operating conditions in yaw that yielded both attached and separated flows over the blades. The derived free-wake geometry solutions are plotted together with the corresponding wake trailing and shed circulation distributions. These plots help investigate how the unsteady bound circulation formed at the blades is eventually convected into the wake. The derived results are helpful to develop more reliable aerodynamic models for wind turbine design codes.

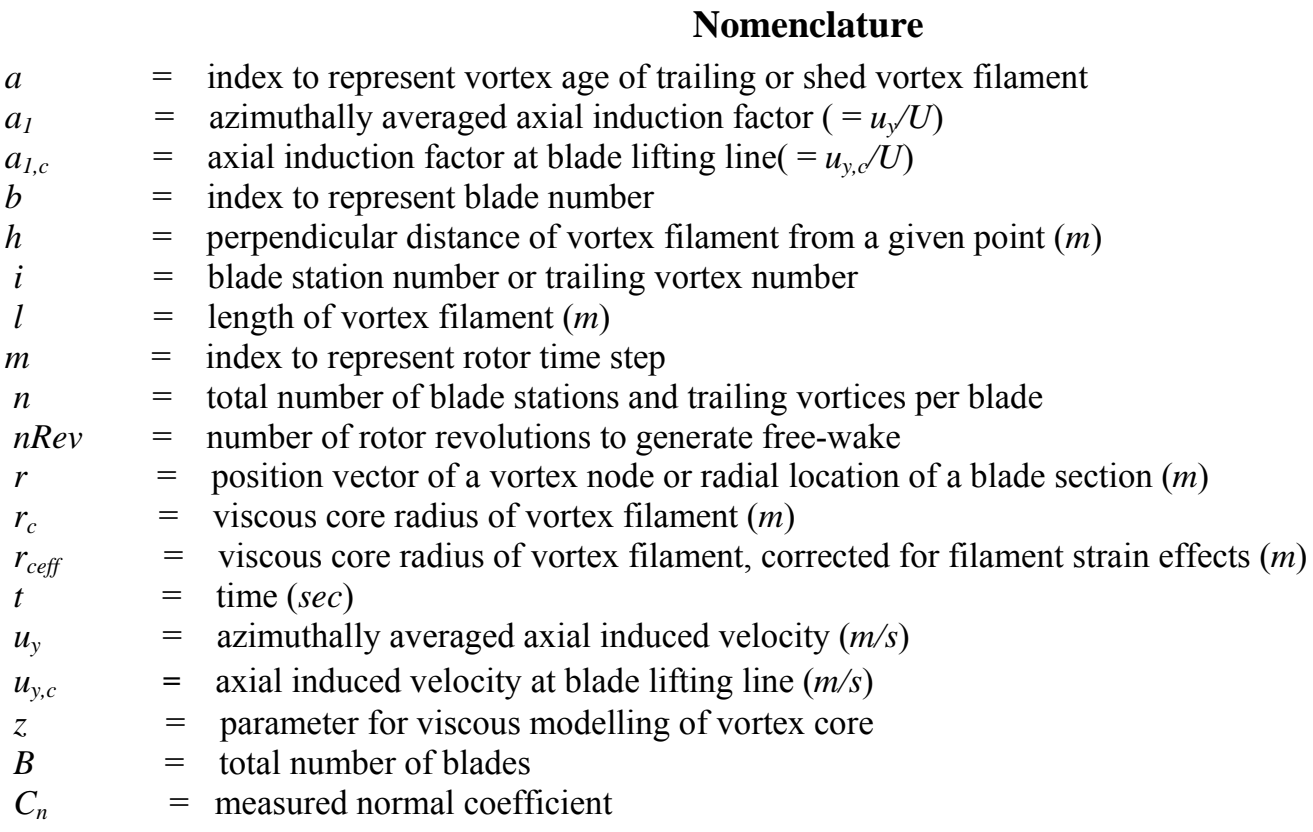

${ }^{*}$ PhD-student, Faculty of Aerospace Engineering, Kluyverweg 1, Delft, The Netherlands, AIAA-member.

${ }^{\dagger}$ Professor, Faculty of Aerospace Engineering, Kluyverweg 1, Delft, The Netherlands, No member.

* Associate Professor, Faculty of Aerospace Engineering, Kuyverweg 1, Delft, The Netherlands, No member. 


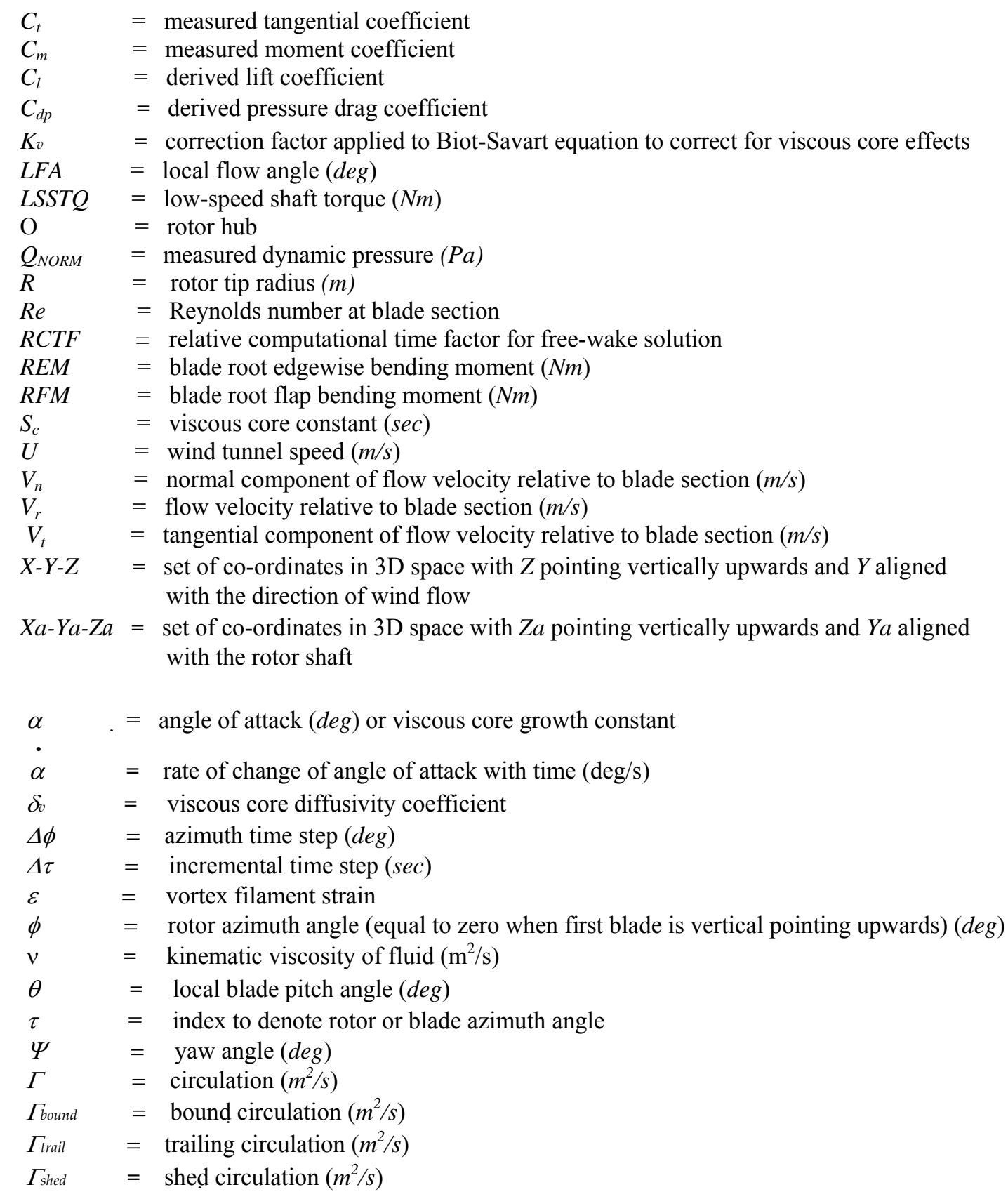

\section{Introduction}

T $\mathrm{N}$ the real operating environment of a horizontal-axis wind turbine, the direction of the wind changes frequently with respect to the rotor axis. As a result of this, the turbine may operate in yaw for considerable amounts of time. The yawed condition introduces a cyclic angle of attack at the blades and causes the helical wake behind the rotor to become skewed, yielding a time-dependent and complex induced velocity distribution at the rotorplane. When the unsteady angle of attack at a blade section exceeds the aerofoil's stalling angle, dynamic stall takes place causing the unsteady aerodynamic loads to be much higher than those predicted by 2D static aerofoil data. The hysterisis effects introduced by dynamic stall may have a negative effect on the aeroelastic damping behavior of wind turbine blades. This reduces the fatigue lifetime leaving an adverse impact on the economics of the system.

To date, the Blade-Element-Momentum (BEM) theory is still the most commonly used method for computing aerodynamic loads during the aeroelastic design of wind turbine blades. Although this theory is suitably accurate for modeling axial conditions provided that reliable aerofoil data is used, it is unreliable when treating unsteady 
conditions associated with yaw. In the past years, several engineering models were added to BEM codes to improve their accuracy in predicting the aerodynamic loads for yawed wind turbine rotors. These models fall under two classifications:

- Type I models: those that correct the standard 2D aerofoil data for 3D effects (stall-delay) and unsteady aerodynamic effects, (unsteady aerofoil models for both attached flow and dynamic stall). Examples of models used to correct for 3D effects are described in Refs. 1, 2, 3. Examples for unsteady aerofoil models used in attached flow conditions are Theordorsen's model ${ }^{4}$ and Leishman's indicial response method using Duhamel's superimposition (Ref. 5, pp 336-340). Examples of dynamic stall models include the ONERA model, BoeingVertol model and the Beddeos-Leishman model. A description of these models may be found in Leishman ${ }^{5,6}$. Most unsteady aerofoil models for attached flow conditions have been derived based on 2D non-rotating wings and therefore there may be inaccurate when applied for $3 \mathrm{D}$ conditions on a rotating wind turbine blade. Several dynamic stall models are semi-empirical and were derived from 2D non-rotating wing experiments. Consequently they are also inaccurate when treating 3D dynamic stall on a rotating blade. Due to the blade advancing-and-retreating effect resulting from yaw, each rotating blade is subjected to unsteady radial flow components that may be much larger in magnitude than in a non-yawed rotor. Such radial flows influence the dynamic stall behavior significantly, as described in Ref. 7. Although it is a well known fact that radial flow over the blades helps in preventing flow separation over the blades at high angles of attack and thus contributes to stall delay, it is still unclear how $3 \mathrm{D}$ effects influence stall in an unsteady environment.

- $\quad$ Type II models: those that correct the axial induction factor computed by BEM for the uneven axial induced velocity distribution at the rotorplane resulting from the skewed wake. An early model for skewed wake effects has been proposed by Glauert ${ }^{8}$ has the form

$$
a_{1, c}=a_{1}\left(1+K \frac{r}{R} \operatorname{Sin}(\phi)\right)
$$

where $K$ is a parameter that depends on the yaw angle. Detailed inflow measurements on the Delft wind tunnel turbine and computations using the more-advanced free-wake vortex model revealed that this model is inaccurate because it only considers the induction from the trailing blade tip vorticity and ignores that contributed by the root vorticity ${ }^{9,10}$. Other models were developed in the past years, some of which were developed in the JOULE Dynamic Inflow projects ${ }^{11,12}$ and have been implemented in various BEM-based aeroelastic models. Although these models improved loads predictions, considerable improvement is still required. This was noted during the "blind comparison" investigation organized by National renewable Energy Laboratory (NREL) way back in the year $2000^{13,14}$. Considerable discrepancies between predicted and experimental results were observed even at low windspeeds at which the angle of attack is small.

Detailed surface pressure measurements on a rotating blade of a yawed wind turbine rotor are very useful in obtaining a deeper understanding of yaw aerodynamics. Such measurements were taken on the NREL Phase VI rotor when tested in the NASA Ames $80 \mathrm{ft} X 120 \mathrm{ft}$ wind tunnel in year 2000. The pressures were integrated along the blades to yield the local blade normal and tangential aerodynamic loads. This data is being analyzed by various

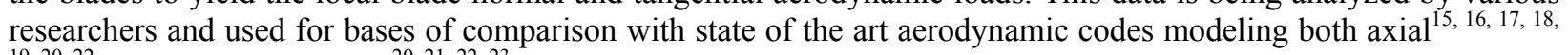
$19,20,22$ and yawed conditions ${ }^{20,21,22,23}$.

A major difficulty with analyzing experimental measurements is to determine an accurate method of finding the angle of attack. This is required to be able to derive the local aerofoil $C_{l}$ and $C_{d}$ from the measured $C_{n}$ and $C_{t}$, as illustrated in figure 1. In the NREL Phase VI experiments, flow direction probes were installed at different radial locations of one blade, just infront of the leading edge to measure the local inflow angle. Due to the influence of the bound circulation at the blades and the wake circulation, the inflow angle may differ significantly from the angle of attack. A correction has to be then used to estimate the angle of attack from the inflow angle, as discussed by Rooj et. al. ${ }^{24}$. Due to the complex flow field across the rotor, the correction that

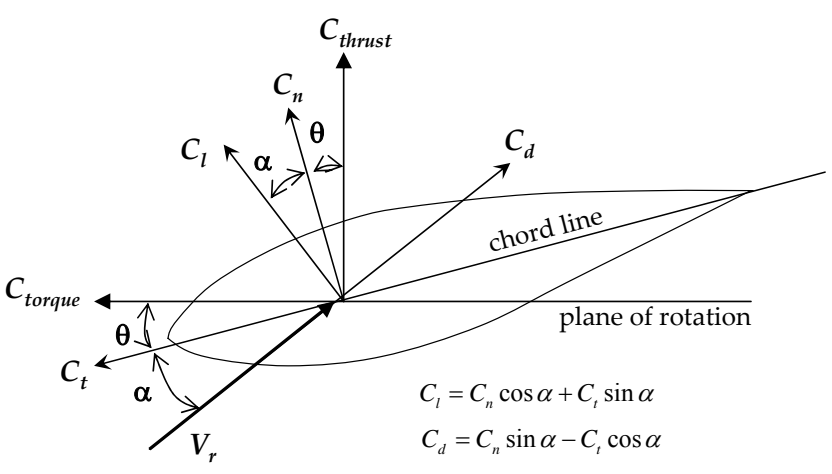

Figure 1 - Blade section aerodynamic load coefficients 
is usually obtained from simple 2D wind tunnel calibration procedures is unreliable. When dealing with yawed conditions, the influences of the unsteady shed vorticity and the effects resulting from the skewed wake will make the required correction very difficult to establish.

Tangler et. al. ${ }^{25,26}$ have adopted an approach in which a prescribed-wake vortex model was used iteratively to derive the angle of attack distributions from the measured $C_{n}$ and $C_{t}$ values of the NREL rotor in axial conditions. In this approach, an initial angle of attack distribution is initially assumed at the blades. This is used to find $C_{l}$ from $C_{n}$ and $C_{t}$. An estimate for the bound circulation at the blades is then made using the Kutta-Joukowski law. This is used to derive the circulation distribution in the prescribed wake, from which the induction at the blades and a new angle of attack is then obtained. This process is repeated until convergence in the angle of attack is achieved.

The objective of this paper is to use a similar approach to derive the angle of attack however applying it for yawed conditions and using a new free-wake vortex model developed at Delft University of Technology. For axial conditions, a prescribed wake vortex model can be reasonably accurate in representing the helical wake that forms downstream of the rotor. However, for yawed conditions the resulting complex skewed wake geometry is more difficult to prescribe. In this sense, a free-wake vortex model would be much more adequate to use since the modeled wake is allowed to develop freely under the action of the wake induced velocities.

In this study the unsteady angle of attack distributions are derived for different operating conditions in yaw of the NREL rotor. These are then used to obtain the aerofoil data for both unsteady attached flow and dynamic stall conditions. This aerofoil data is then compared with that obtained for axial conditions as well as with the 2D wind tunnel data for the same aerofoil. From the free-wake plots, the time-dependent induced velocity distributions at the rotorplane are computed and analyzed. Using the derived aerofoil data and induced velocity distributions, the rotor aerodynamic loads could be calculated and compared with those measured during the wind tunnel experiments. Finally, the free-wake model is also used to estimate the inflow angles at the measuring tip locations of the flow direction probes. These are then compared with those obtained in the experiments. The results of this study provide further insight into the aerodynamics of rotors in yaw and could be helpful in developing better aerodynamic models to be integrated into aeroelastic design codes.

\section{NASA Ames UAE Wind Tunnel Data Used}

The NASA Phase VI rotor is a two-bladed 10m diameter wind turbine rotor. The geometry of the blades is based on the S809 aerofoil. Details about the blade design may be found in Ref. 27. One of the blades was equipped with pressure sensors at 5 radial locations: $0.30 R, 0.47 R, 0.63 R, 0.80 R$ and $0.95 R$ to measure the surface pressures. The blade was also equipped with five-hole pressure probes at $0.34 R, 0.51 R, 0.67 R, 0.84 R$ and $0.91 R$ to measure the local inflow angle (LFA). At each of these radial locations, the surface pressures were integrated to obtain $C_{n}, C_{t}$ and $C_{m}$. Strain-gauge techniques were used to measure the low-speed shaft torque and the blade root flap and edge moments at one of the blades. Further information about these UAE experiments is available in Refs. 13,28. The turbine was tested over a wide range of operating conditions. This study only considered axial conditions and yaw at 30 degrees for wind speeds 5,10 and $15 \mathrm{~m} / \mathrm{s}$ with the rotor operating in the upwind position. Yaw angles above $30^{\circ}$ were not included in this study since their measurements were expected to be considerably affected by the flow disturbances from the box/boom installed upstream of the rotor (refer to reference 28). The blade pitch and rotor coning angle were maintained constant at $3^{0}$ and $0^{0}$ respectively. This study only considers the experimental data collected with the ' $\mathrm{S}$ ' and ' $\mathrm{H}$ ' configurations (refer to reference 28 ).

For each operating condition, data records of the surface pressures were taken for 36 individual rotor rotations (cycles). Fig. 2 shows typical variations for $C_{n}$ obtained at each individual cycle at $0.3 R$ at yaw 30 degrees and a wind speed of $15 \mathrm{~m} / \mathrm{s}$. In this figure, data from five different cycles are shown. It may be observed that for blade azimuth angles between approximately $120^{\circ}$ and $240^{\circ}$, the cycle-to-cycle variation is very small. As it will be noted later on in the paper, for this region of blade azimuth angle, the angle of attack is small and the flow is attached. For the other blade azimuth angles, the cycle-to-cycle variation is large and this is due to the high angles of attack were flow separation influences reduce the repeatability of the measured data. In all cases, the mean (indicated by the solid black line in Fig. 2) is considered for the analysis with the free-wake code. 


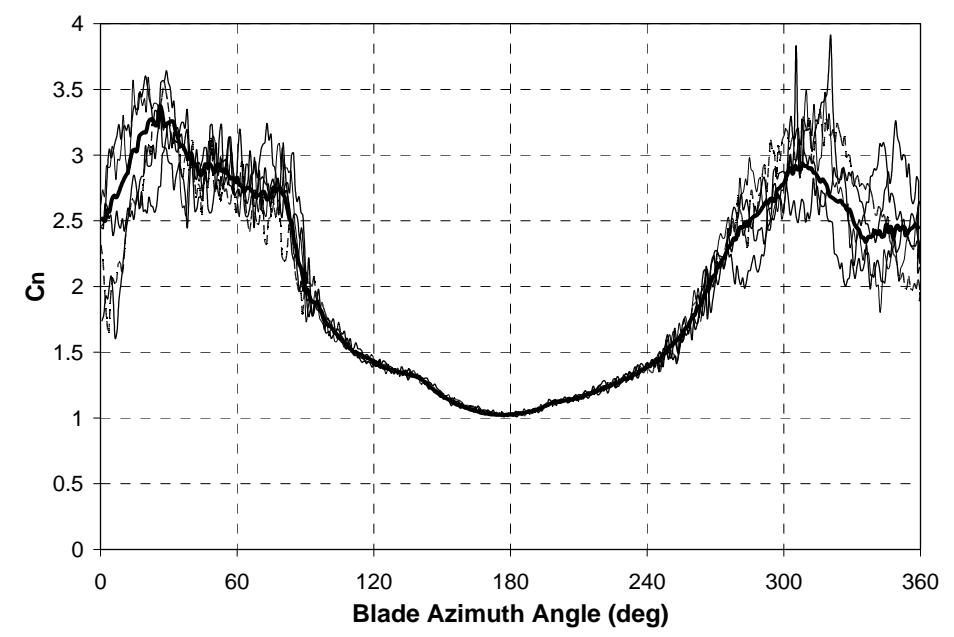

Figure 2 - Variation of the normal force coefficient with blade azimuth angle for Yaw $30 \mathrm{deg}, U=15 \mathrm{~m} / \mathrm{s}$ and $r / R=0.3$. Values for five separate cycles are shown and the cycle mean is indicated by the bold curve.

\section{Free-Wake Vortex Model}

The subject free-wake vortex model was specifically designed to simulate rotor wakes from knowledge of the aerodynamic loads at the blades. Unlike other free-wake vortex models, it does not directly rely on the availability of aerofoil data to iteratively determine the blade loading. The bound circulation distribution needs to be prescribed as input. From this prescription, the code generates a free-wake and then calculates the $3 \mathrm{D}$ induced velocities at different points in the flow field of the rotor. The principle used for this model is based on the fact that, assuming inviscid and incompressible flow conditions, the circulation in a flow field is conserved. Thus from a time-history of bound circulation at the blades, it is possible to derive the $3 \mathrm{D}$ circulation distribution in the wake.

\section{A. Blade Model}

Each rotor blade is modeled by a lifting line with a single lumped vortex located along the quarter-chord point of the blade sections. A cosine radial segmentation for the lifting line is used to enhance numerical accuracy, especially towards the blade tip and root, where the bound circulation is known to vary rapidly with radial position.

\section{B. Wake Model}

The wake is modeled as two different parts: a near wake and a far wake.

Near wake: The near wake is the actual free-wake and is made up of vortex sheets, one for every blade. Each vortex sheet consists of a lattice of straight-line vortex filaments to represent the trailing and shed vorticity in the wake (Fig. 3). The filaments are interconnected by nodes whose positions are updated after each rotor time step. To satisfy the Kutta condition, each node leaves from the blade's trailing edge, from where it travels downstream with a local velocity that is equal to the vectorial sum of the windspeed and the vorticity induced velocity. The latter is calculated by applying the Biot-Savart equation.

Viscous effects in rotor wakes usually occur at much smaller scales than potential flow phenomena. However, these effects may be significant in yawed conditions due to the formation of complex and unsteady blade-to-wake interactions and skewed wake effects. In this free-wake code, viscous effects are accounted for using an approach very similar to Leishman et al. ${ }^{29}$ in which a viscous parameter $K_{v}$ is introduced in the Biot-Savart law as follows:

$$
d \vec{u}=K_{v} \frac{\Gamma}{4 \pi} \frac{d \vec{s} \times \vec{r}}{|\vec{r}|^{3}}
$$

where

$$
K_{V}=\frac{h^{2}}{\left(r_{c}^{4}+h^{4}\right)^{1 / 2}}
$$




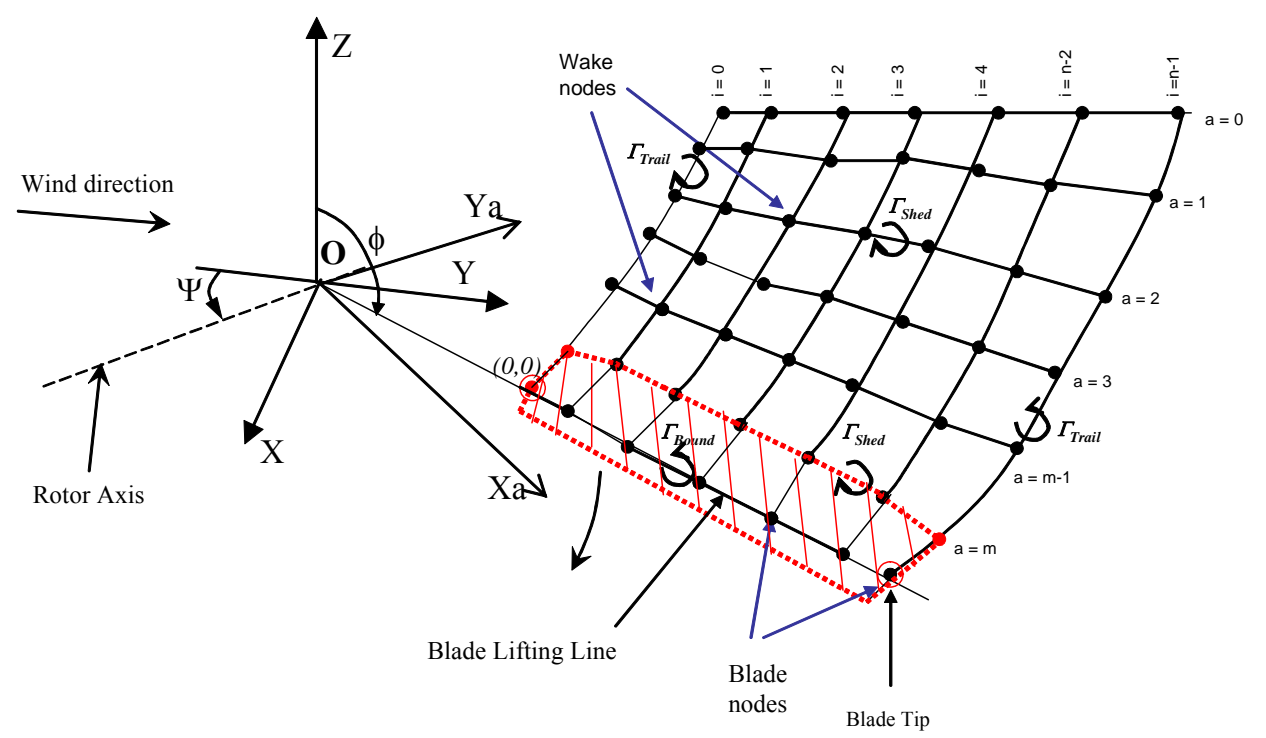

Figure 3: Modeling of blades and near wake in free-wake vortex model

Equation (3) is the Lamb-Oseen ${ }^{29,30}$ viscous core model in which the inner part of the vortex, denoted as the core region $\left(r<r_{c}\right.$ ), almost rotates as a solid body. The outer region (outside the core, $r>r_{c}$ ) almost behaves as a potential flow. The viscous diffusion of vorticity in this free-wake model is handled by applying a core growth model. A core growth model was adopted from Ananthan $e a^{31}$ and is applied to every trailing and shed vortex filament. The one-dimensional laminar Navier-Stokes equations reveal that the viscous vortex core radius grows with time in accordance with ${ }^{5,29,30,31}$ :

$$
r_{c}(t)=\sqrt{4 \alpha \delta_{v} v t}
$$

where $\alpha$ is a constant equal to 1.25643 . $\delta_{v}$ is the turbulent viscosity coefficient that is added to Eq. (4) by Leishman (Ref. 5, pp 441) to account for the increased vorticity diffusion in turbulent flows. Experimental investigations ${ }^{30,31}$ suggest that $\delta_{v}$ is about 10 for small-scale rotors and is higher for full-scale rotors (on the order 100 to 1000). Current research intends to develop relations for $\delta_{v}$. In Eq. (4), $t=0$ refers to the moment at which a vortex is shed from the blade's trailing edge. Due to the formation of a boundary layer on the blade surfaces, viscous effects are present at the trailing edge. Consequently, each vortex sheet in the free-wake model should therefore have some thickness here within which viscous effects are considerable. The following relation is used in the subject free-wake code to increase the core radius gradually with time:

$$
r_{c}(t)=\sqrt{4 \alpha \delta_{v} v\left(t+S_{c}\right)}
$$

$S_{c}$ is a time-offset parameter that sets a non-zero viscous core radius for vortices that are just released from the trailing edge of each blade. In a free-wake solution the wake nodes are allowed to convect freely, thereby causing the vortex filaments to be strained. This results in a change in the vorticity content of the individual filaments which in turn modifies the induced velocity field around the vortex core. To account for filament straining, the core radius estimated by Eq.(5) is corrected using:

$$
r_{c_{\text {eff }}}=r_{c_{o}}\left(\frac{1}{\sqrt{1+\varepsilon}}\right)
$$

where $\varepsilon$ is the vortex filament strain. The derivation of Eq.(6) may be found in Ref. 31 and is based on Helmotz's third law stating that the net strength of a vortex should remain constant.

Far-wake: the far-wake model consists of a helical tip vortex at each blade, representing fully rolled up tip vortices. The helices extend downstream with prescribed pitch, number of rotations and wake skew angle. Each helix has a constant diameter and starts from the respective near wake node with co-ordinate $(0, n-1)$, refer to Fig. 3 . In yawed conditions the helices are skewed such that their central axis is aligned with the wake skew axis. The helices are segmented into straight-line vortex filaments and the standard Biot-Savart law Eq.(2) is applied with a simple numerical cut-off method. Since in a yawed rotor, the bound circulation at the blades is a function of azimuth angle, then the circulation assigned to each filament is varied, depending on its azimuth position on the helix. Each filament is assigned to the maximum bound circulation that occurs on the blade when the latter is at the same azimuth position as that of the filament. 


\section{Numerical Solution}

Each rotor revolution is divided into a fixed number of equally-spaced azimuth positions. The bound circulation distribution is prescribed at each blade for each azimuth position. The viscous modeling parameters $\left(\delta_{v}\right.$ and $\left.S_{c}\right)$ are also prescribed and these remain constant throughout the whole solution. A time-marching approach is adopted with the solution started with an impulsive start of the rotor. Initially, the first blade is set at the zero azimuth position (blade vertical pointing upwards) and there is no near wake, but only the prescribed far wake. The rotor then starts rotating at constant speed. At each time step, trailing and shed vortex filaments are shed from the blades' trailing edges with a circulation calculated from the spanwise and time variations of the local bound circulation. This will eventually form a near wake that will extend downstream. At each time step, the core size of each vortex each calculated from Eq.(5) and corrected for strain in accordance with Eq.(6). The 3D induced velocities at each wake node due to all bound, trailing and shed vortices are computed using the modified Biot-Savart equation Eq.(2). The absolute velocity of the wake nodes is then estimated by vectorially adding the flow free steam velocity and the local induced velocities. Before moving to the next time step, the position of the wake nodes is updated using an Euler explicit time-integrating scheme. To improve the accuracy and stability of the numerical solution, the absolute velocity used in this scheme is taken to be equal to the average of the current absolute velocity and that of the previous time-step. The above process is repeated until the rotor has rotated the required number of revolutions (nRev). During the final revolution, the Biot-Savart law Eq.(2) is used to compute the induced velocity across the whole rotor plane (or at any other plane parallel to rotorplane) at different rotor azimuth positions.

\section{Methodology}

\section{A. Computational Procedure used to derive the Unsteady Angle of Attack using the NREL Experimental Data and the Free-wake Model}

Fig. 4 illustrates the procedure used to derive iteratively the unsteady angle of attack distributions from the NREL experimental data using the free-wake vortex model. The experimental data was only available for one blade. The same data is applied for the other blade, obviously taking into account the respective blade azimuth angles. The computational procedure consists of the following steps:

Step 1: The measure values of $Q_{\text {NORM }}, C_{n}$ and $C_{t}$ at the different radial locations and blade azimuthal angles were first loaded into the code from the NREL experimental database (' $S$ ' configuration ${ }^{28}$ ).

Step 2: An angle of attack distribution was then assumed and used in equation shown in Fig. 1 to obtain an estimate for $C_{l}$ at the different radial locations and blade azimuthal angles.

Step 3: The relative flow velocity, $V_{r}$, was found from $Q_{N O R M}$ using the equation for dynamic pressure given by

$$
Q_{\text {NORM }}=\frac{1}{2} \rho V_{r}^{2}
$$

Step 4: The Kutta-Joukowksi theorem for a lifting line is used to find the bound circulations at the pressure measurement stations:

$$
\Gamma_{B}=\frac{1}{2} C_{l} V_{r} C
$$

Step 5: A double interpolation was made to obtain a bound circulation at the blades as a function of radial position and blade azimuth position $\left(\Gamma_{B}[r / R, \phi]\right)$, with the bound circulation at the blade tip and root set to zero. For interpolating, a spline interpolation was initially used to obtain $\Gamma_{B}$ as a function of $r / R$. But it was found that the number of pressure measuring stations along the blade (equal to 5) was not enough to give an accurate representation of the bound circulation distribution along the blade with a spline method. This caused the spline method to yield unrealistic 'jumps'. Alternatively, a linear interpolating method had to be adopted. A spline interpolation was used only to interpolate for different blade azimuth positions.

Step 6: the interpolated bound circulation distribution $\left(\Gamma_{B}[r / R, \phi]\right)$ was then prescribed to the free-wake code and the modeled rotor was set to rotate to generate the free-wake extending downstream for a given number of rotations. This was used to calculate the unsteady $3 \mathrm{D}$ induced velocities at the lifting line of each rotor blade from which a new angle of attack estimate is obtained using the equation derived from Fig. 1:

$$
\alpha=\tan ^{-1}\left(V_{n} / V_{t}\right)
$$

Step 7: the new angle of attack was used in Eq.(8) to yield a new bound circulation distribution and the whole process is repeated until convergence in the angle of attack is at all radial locations and rotor azimuth angles. 
Once a converged solution was obtained, the final distributions of the unsteady values of $C_{l}$ and $C_{d p}$ were derived in accordance with Fig. 1. From knowledge of the angle of attack variations with rotor azimuth angle, it was possible to derive the time-rate of change of angle of attack, together with the hysterisis loops for $C_{l^{-}} \alpha$ and $C_{d p}-\alpha$. The computed free-wake geometries are used to calculate the unsteady induction distributions at the rotorplane. The latter are then used together with the derived lift and drag coefficients to evaluate the rotor/blade loads as a function of rotor azimuth angle. These loads are compared with those measured during the wind tunnel experiments. Finally, using the free-wake vortex model, it was possible to compute the local inflow angles at the measuring tip of the flow direction probes ('H' configuration ${ }^{28}$ ). These were also compared with the corresponding experimental values.

The above procedure was repeated for each of the operating conditions mentioned in section II. In these calculations the flow disturbances due to the tower and nacelle were ignored. Structural dynamic influences were also neglected.

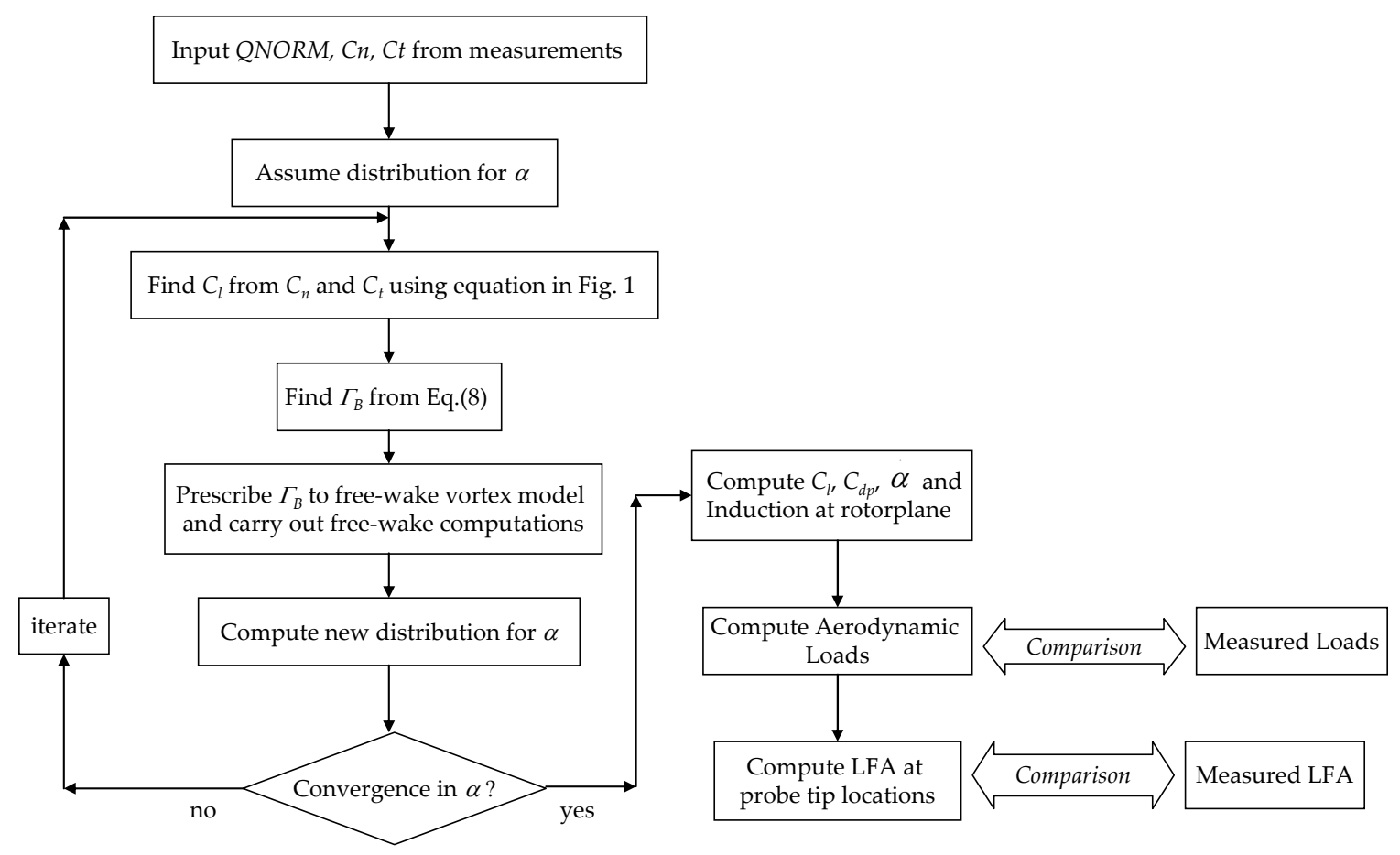

Figure 4 - Computational procedure used to derive the angle of attack from $C_{n}$ and $C_{t}$ using free-wake vortex model

\section{B. Selected Free-Wake Model Parameters}

In all free-wake computations, each blade was divided into 21 blade sections while the azimuth step size was taken to be equal to 36 steps per revolution. These values were found to be sufficient to minimize the errors due to the numerical solution. In selecting the required value of $n R e v$, two conditions were used: Condition 1 is that the induced velocity at the rotorplane is independent of $n R e v$; Condition 2 is that the induced velocity at the rotoplane due the far wake is negligible compared with that due to the near wake. To satisfy Condition 1, wake periodicity should be achieved such that the induced velocity at the blade lifting line of the first blade at an azimuth angle of $360^{\circ}$ should be equal to that at an azimuth angle of $0^{0}$. The discrepancy is expressed here as

$$
\xi_{w p}=\frac{\left.u_{y, c}\right|_{\phi=0^{0}}-\left.u_{y, c}\right|_{\phi=360^{0}}}{\left.u_{y, c}\right|_{\phi=360^{0}}} \times 100
$$

To satisfy both conditions, nRev had to be varied according to the windspeed. nRev was taken to be equal to 5,4 and 3 at windpeeds $U=5,10$ and $15 \mathrm{~m} / \mathrm{s}$ respectively. The near wake extended downstream by $3.6 R, 6 R$ and $7 R$ 
respectively. At these distances the values of $\xi_{w p}$ at all radial locations were $<1.5 \%$, while the axial induction at the lifting lines due to the far wake alone was $<3 \%$ of the total axial induction.

As regards the far wake parameters, the azimuthal step and number of helical revolutions were kept constant at $10^{\circ}$ and 10 while the prescribed helical pitch and wake skew angle were varied depending on windspeed. To determine reasonable values for the prescribed helical pitch and wake skew angle at each windspeed, preliminary calculations were carried out with the same free-wake model and an estimate was obtained from the near wake geometry.

It should be appreciated that, considering the limitations of vortex models to cater for viscous effects, it is very difficult to select the optimum values for $\left(\delta_{v}, S_{c}\right)$. This is even more difficult when modelling yawed conditions in which viscous effects become subject to dynamic flow influences. Due to this difficulty, an analysis was therefore carried out in this study to investigate the sensitivity of $u_{y, c}$ to different values of $\left(\delta_{v}, S_{c}\right)$. The analysis was performed at two windspeeds only ( 7 and $13 \mathrm{~m} / \mathrm{s}$ ) which yielded attached flow and separated flow conditions at the blades, respectively. Four different sets of values of $\left(\delta_{v}, S_{c}\right)$ were used: $(10,0.1),(100,1),(500,1)$ and $(500,10)$. Figures 5 and 6 illustrate the resulting spanwise variations at different blade azimuth positions $(0,120$ and $240 \mathrm{deg})$. It is observed that the sensitivity of $u_{y, c}$ at the first 3 sets of values of $\left(\delta_{0}, S_{c}\right)$ is very small. But a notable relative discrepancy is seen for $(500,10)$. The latter implies that care should be taken in selecting which values of $\left(\delta_{v}, S_{c}\right)$ to assign for the computations, since this could influence considerably the results.

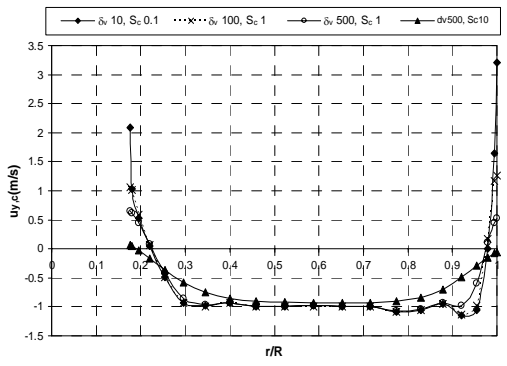

Fig (a): $\phi=0$ deg

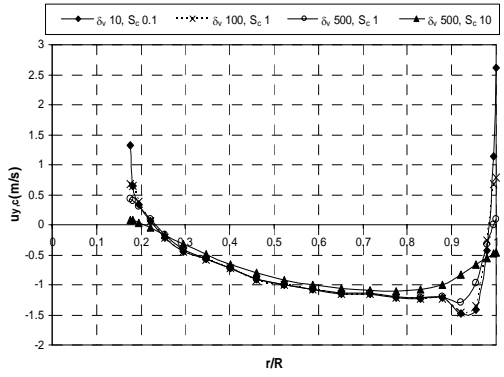

Fig (b): $\phi=120 \mathrm{deg}$

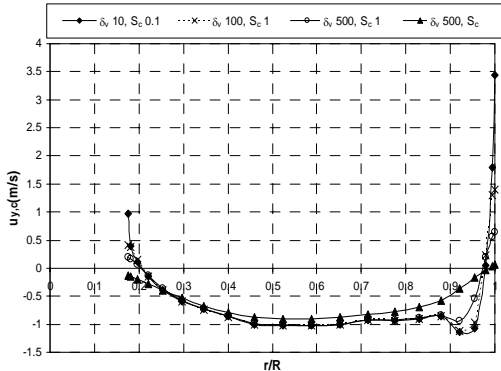

Fig (c): $\phi=240 \mathrm{deg}$

Figure 5 - Effect of viscous parameters $\left(\delta_{v}, S_{c}\right)$ on the spanwise distribution of $u_{y, c}$ at different blade azimuth angles, $U=7 \mathrm{~m} / \mathrm{s}$

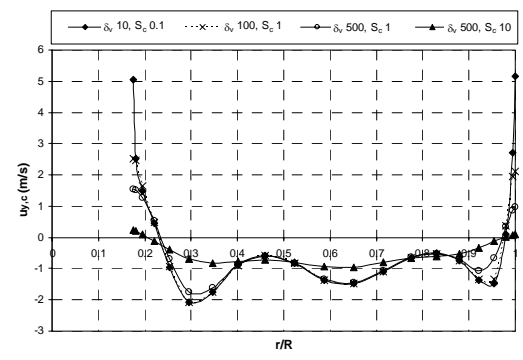

Fig (a): $\phi=O \mathrm{deg}$

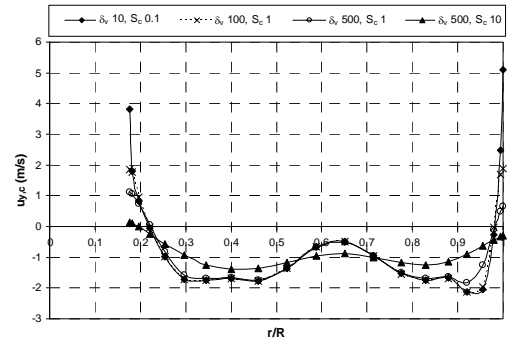

Fig (b): $\phi=120 \mathrm{deg}$

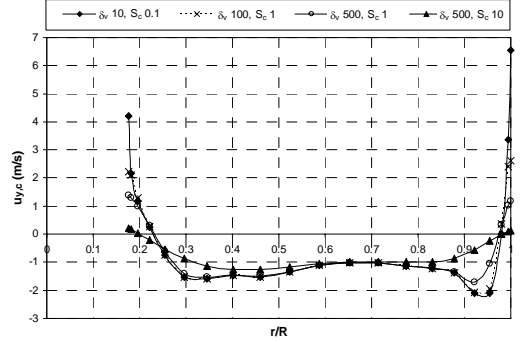

Fig (c): $\phi=240 \mathrm{deg}$

Figure 6 - Effect of viscous parameters $\left(\delta_{v}, S_{c}\right)$ on the spanwise distribution of $u_{y, c}$ at different blade azimuth angles, $U=13 \mathrm{~m} / \mathrm{s}$ 
As it may be noted from Eq.(5), increasing both $\left(\delta_{v}, S_{c}\right)$ will increase the viscous core radius of the trailing and shed vortices leaving from trailing edge of the blades. In attempting to select realistic values for these viscous parameters for this study, it was assumed that twice the value of $r_{c}$ at the blade trailing edge (i.e. at $t=0$ ) should approximately be equal to the thickness of the viscous layer of the wake vortex sheets. This thickness depends on the boundary layers formed on the upper and lower surfaces of the aerofoil which are in turn a function of the local Reynolds number and the angle of attack. For attached flow conditions (low angles of attack), the boundary layers are thin. The blade sections of the rotor at the operating conditions stated in section II have a local Reynolds number in the range $0.6-1.1 \times 10^{6}$. It was estimated using RFIOL that, for this Reynolds number range, the maximum boundary layer thickness at the trailing edge is only $7.82 \times 10^{-3} \mathrm{C}$ up to an angle of attack of $10^{\circ}$. Consequently, the thickness of the viscous layer of the vortex sheet at the blade's trailing edge is also small and it does not exceed the local blade thickness $(20.95 \mathrm{c} \%$ ) in attached flow conditions (Fig. 7). At high angles of attack however, flow separation on the blade's upper surface may cause the thickness of the viscous layer to be larger than the local blade thickness. The maximum angle of attack observed during the subject experiments was about $45^{\circ}$ at $\mathrm{U}=15 \mathrm{~m} / \mathrm{s}$ and $\mathrm{r} / \mathrm{R}=0.3$ (refer to Fig. 9(c)). At this angle of attack, however it is unlikely that the thickness of the viscous layer exceeds the blade chord length, considering also the fact that there are strong coriolis forces at $r / R=0.3$ which tend reduce flow separation influences.

The values of $2 * r_{c}$ at the trailing edge $(t=0)$ predicted by Eq.(5) for the different sets of values of $\left(\delta_{0}, S_{c}\right)$ were compared with the blade thickness (which is equal to 20.95\%c for the S809 aerofoil) and the blade chord spanwise distributions. This comparison is illustrated in Fig. 8. It is shown that parameters $(500,10)$ yield a value of $2 * r_{c}$ that is much larger than the maximum blade chord. This is not realistic. Thus, a lower value of $2 * r_{c}$ was suggested for this study. Values $(500,1)$ tend to be suitable for separated flow conditions since the resulting value of $2 * r_{c}$ is between $20.95 \% c$ and $c$. For attached flow conditions the values $\delta_{v}$ and/or $S_{c}$ should strictly speaking be decreased so as to reduce $2{ }^{*} r_{c}$ to be less than $20.95 \%$ c. However, since this does not effect considerably the calculated distributions of $u_{y, c}$ (refer to Fig. 5), $\left(\delta_{v}, S_{c}\right)$ were set to $(500,1)$ for attached flow conditions also.

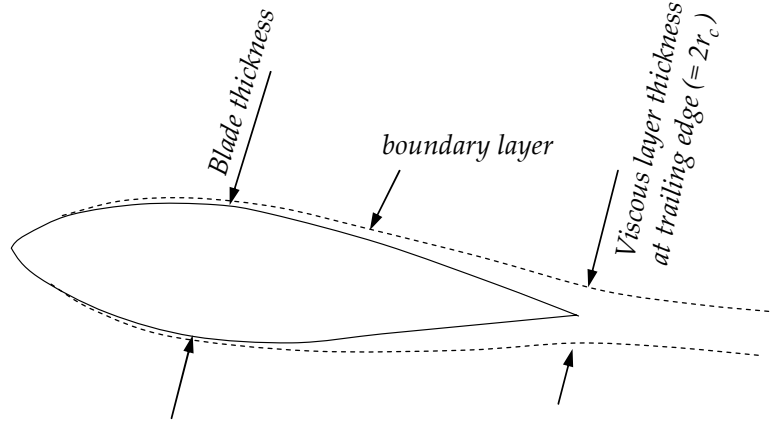

Low angle of attack (attached flow)

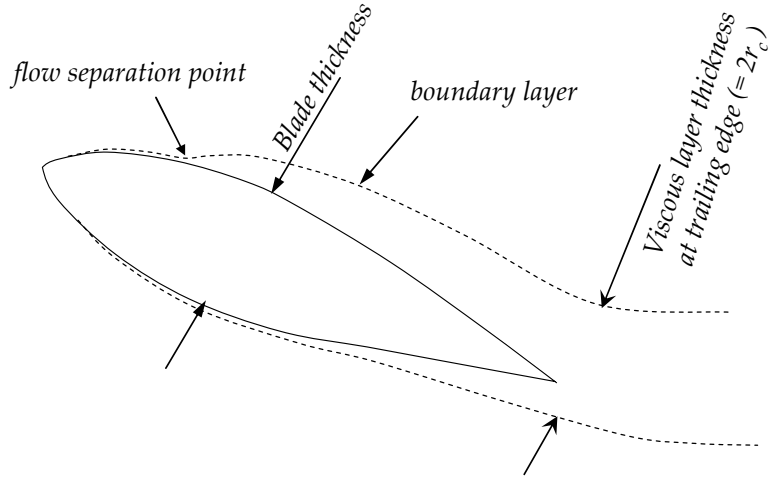

High angle of attack (separated flow)

Figure 7: Influence of the angle of attack on the variation of the viscous layer thickness of wake vortex sheet at trailing edge 


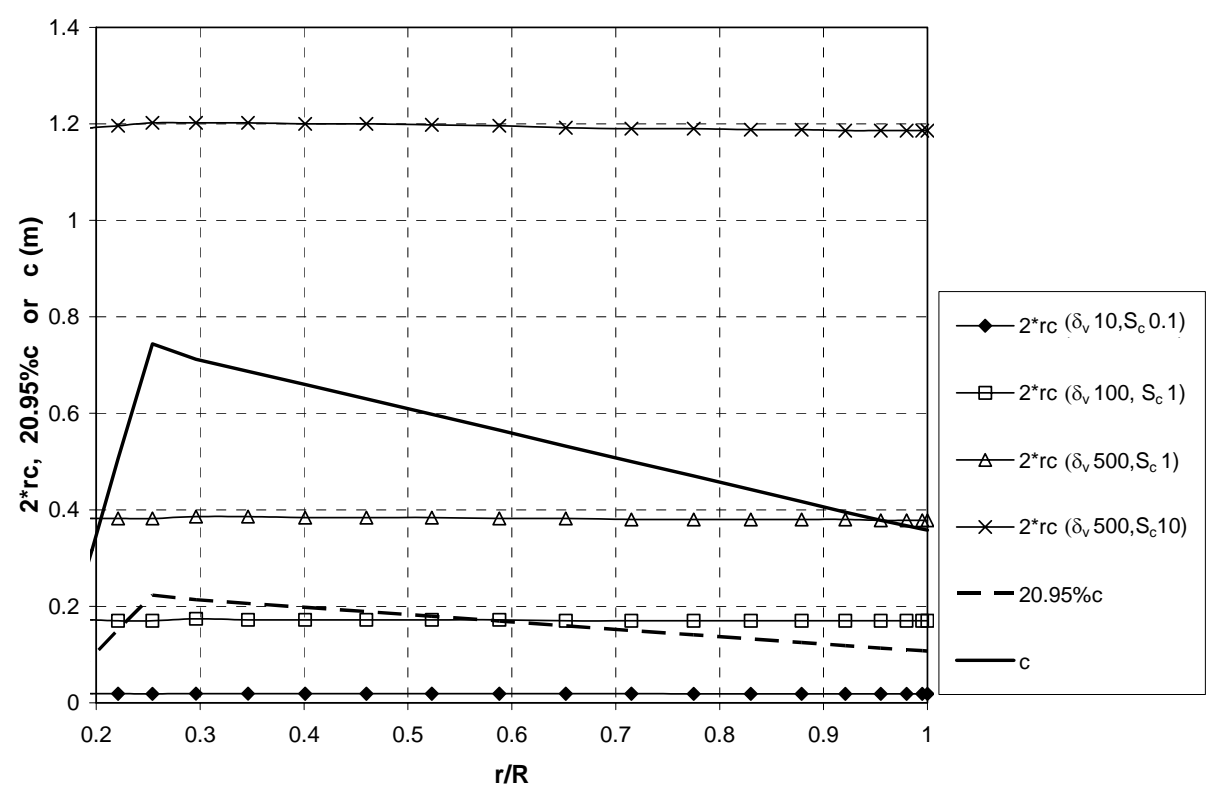

Figure 8 - Comparison of $2^{*}{ }^{*} c$ calculated at different values for viscous parameters $\left(\delta_{0}, S_{c}\right)$ with the spanwise distribution of blade thickness $(30 \% c)$ and blade chord (c)

\section{Results and Discussion}

This section describes the results obtained from a converged solution in the angle of attack from the NREL blade pressure measurements at windspeeds 5,10 , and $15 \mathrm{~m} / \mathrm{s}$ and at a yaw angle of $30 \mathrm{deg}$ using the procedure of section IV A. and free-wake model parameters of section IV B.

\section{A. Variation of $\alpha, \dot{\alpha}, C_{l}$ and $C_{d p}$ with blade azimuth angle $(\phi)$}

Figures 9(a)-(c) illustrate the variation of the angle of attack, the rate of change of the angle of attack, the lift and drag coefficients with the blade azimuth angle at each of the different tunnel wind speeds. It may be observed that, for all windspeeds, the angle of attack varies nearly sinusoidally at all blade radial positions. The angle of attack is the minimum when the blade is approximately at $180 \mathrm{deg}$ position and reaches a maximum when the blade is at 0/360 deg position. For a particular wind speed, both the mean and cyclic components of the angle of attack are larger at the inboard blade sections, implying that the inboard sections experience higher unsteady effects and are first to experience stall. Increasing the windspeed increases both the mean and cyclic components of the angle of attack.

In figures 9(a)-(c) it may also be noted that the time rate of change of angle of attack $(\dot{\alpha})$ varies also nearly sinusiodally with blade azimuth angle at the lower wind speeds $(U=5$ and $10 \mathrm{~m} / \mathrm{s})$. At high wind speeds however $(\mathrm{U}=15 \mathrm{~m} / \mathrm{s})$, the variation does not remain sinusoidal. The rate of change of angle of attack is negative during approx. blade angles 0 to 180 degrees while it is positive from about 180 to 360 degrees. The inboard blade sections tend to experience the highest rates of change in the angle of attack, occurring at approximately blade angles 90 and 270 degrees. Increasing the windspeed while keeping the rotor shaft speed constant results in higher values for $\dot{\alpha}$. At $\mathrm{U}=5 \mathrm{~m} / \mathrm{s}, \dot{\alpha}$ at $\mathrm{r} / \mathrm{R}=0.3$ is equal to about $+/ 25 \mathrm{deg} / \mathrm{s}$ while at $\mathrm{U}=15 \mathrm{~m} / \mathrm{s}$, this reaches maximum values of about $+/-$ 200deg/s.

As regards to the variation of the lift coefficient with blade azimuth angle, it may be observed that, at each windspeed, the inboard regions of the blades experience higher values in both the mean and cyclic components of $C_{l}$. At low windspeeds $(5$, and $10 \mathrm{~m} / \mathrm{s})$, the variation of $C_{l}$ with $\phi$ is sinusoidal reaching a maximum value at blade position $0 / 360 \mathrm{deg}$ and a minimum value at about 180 degree. At the higher wind speeds, $(U=15 \mathrm{~m} / \mathrm{s})$, the variation is no longer sinusoidal. In general, the inboard sections experience higher cyclic lift coefficient values.

The pressure drag coefficients $\left(C_{d p}\right)$ are very small at low wind speeds $(\mathrm{U}=5 \mathrm{~m} / \mathrm{s})$. But as the windspeed is increased, the pressure drag coefficient values increase rapidly, the highest values being at the inboard blade sections at blade angles 0-120 deg and 240-360 deg. 

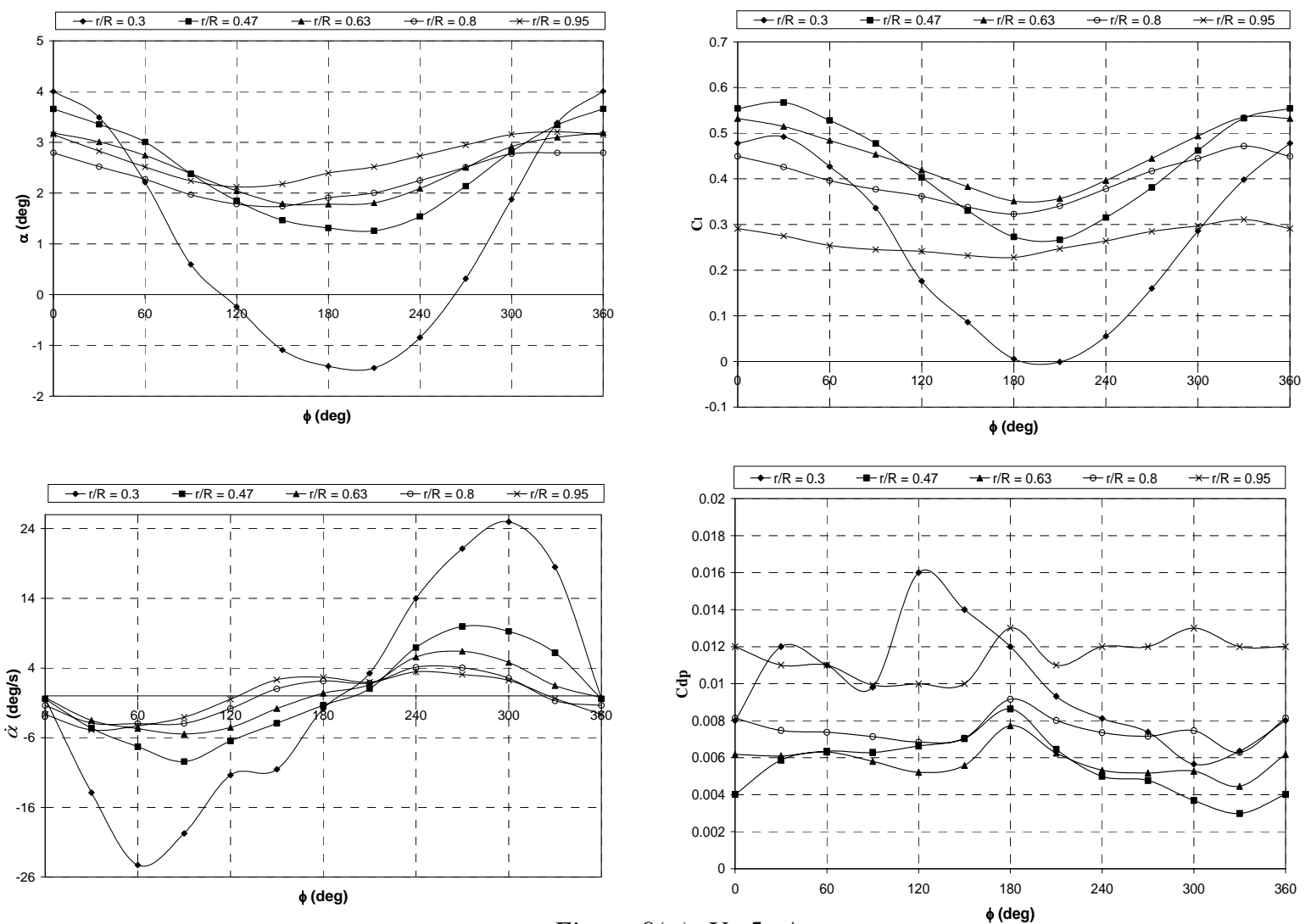

Figure 9(a): $U=5 \mathrm{~m} / \mathrm{s}$
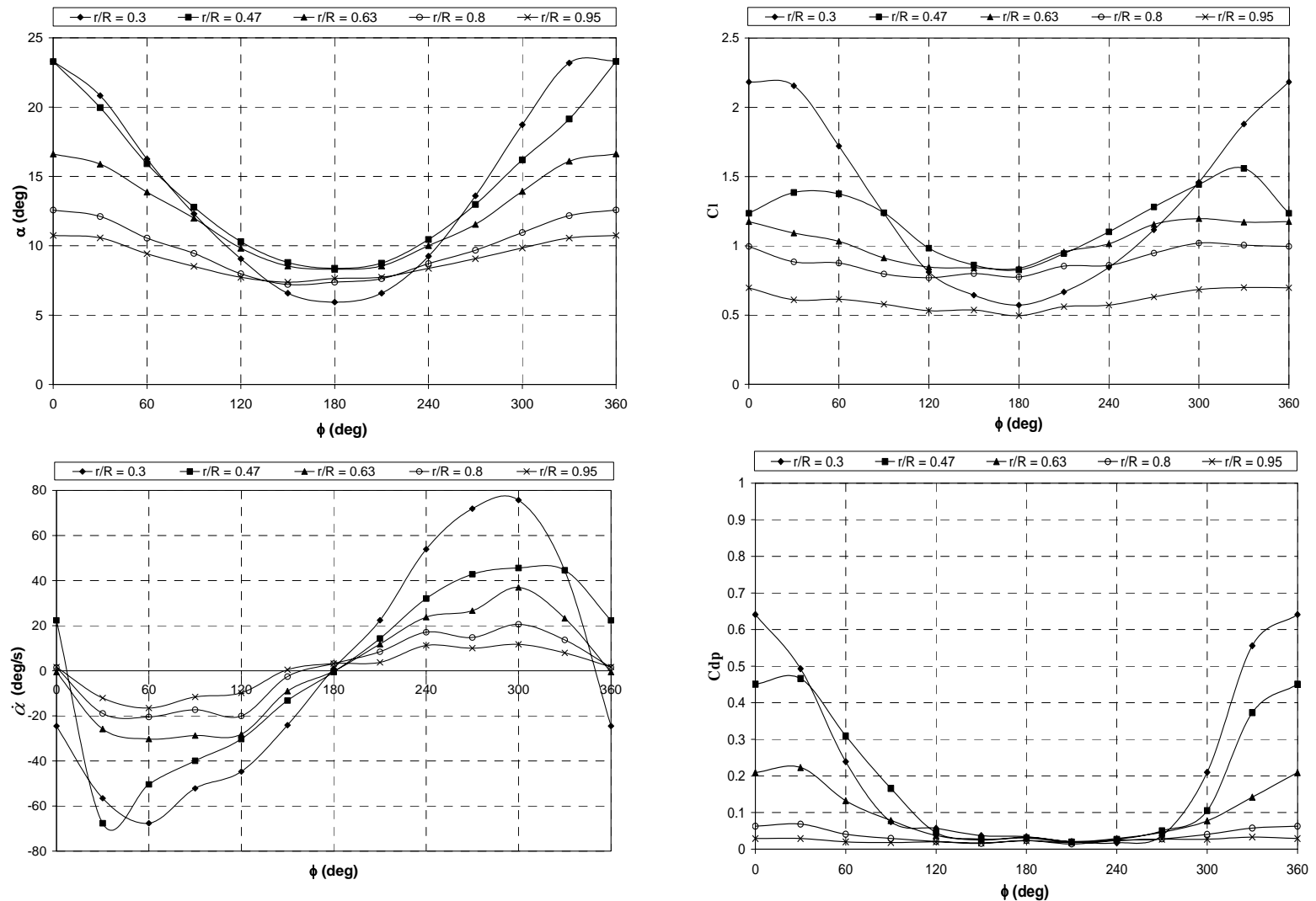

Figure 9(b): $U=10 \mathrm{~m} / \mathrm{s}$ 

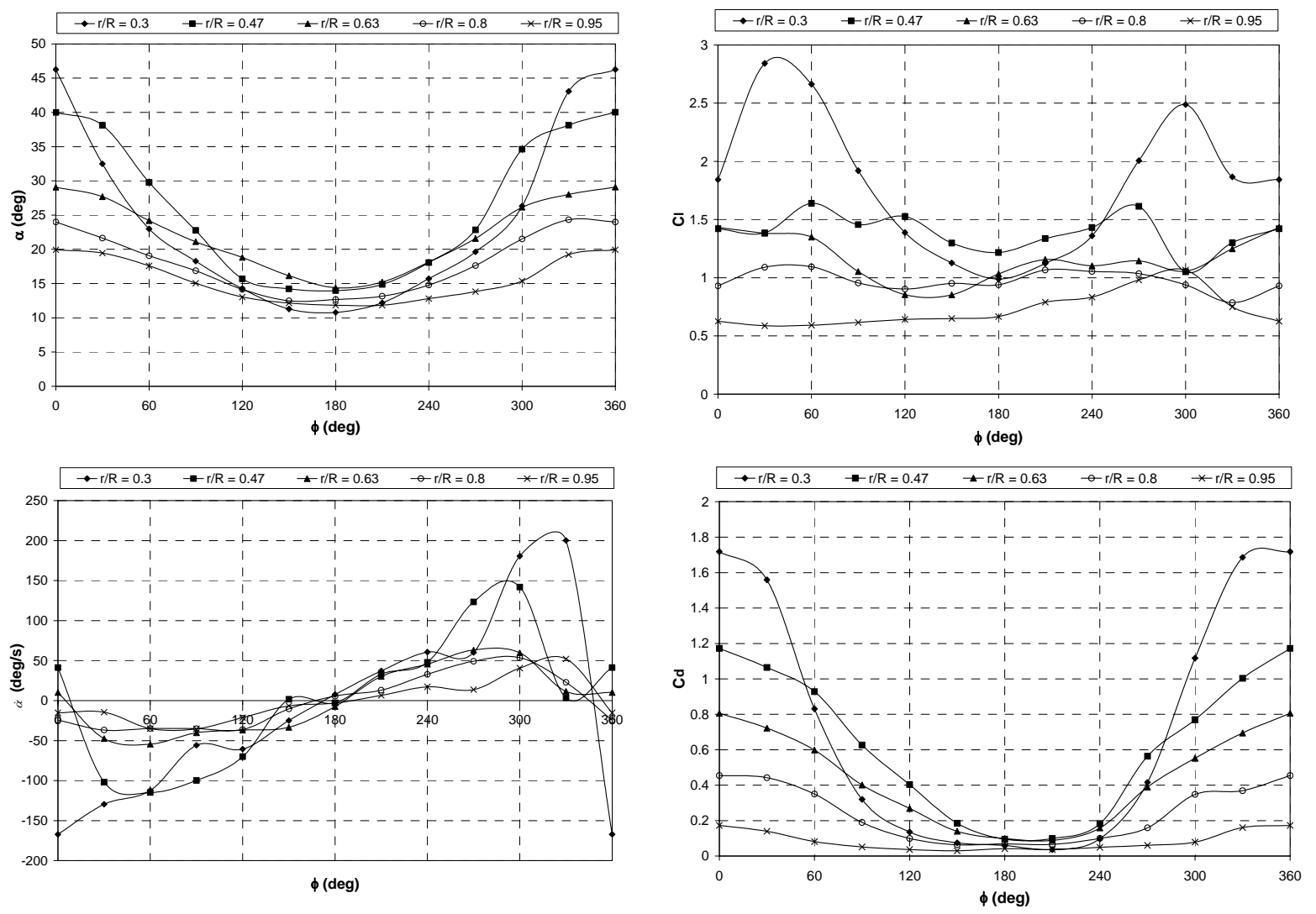

Figure 9(c): $U=15 \mathrm{~m} / \mathrm{s}$

\section{B. Hysterisis loops $C_{l^{-}} \alpha$ and $C_{d^{-}} \alpha$}

From the data presented in figures 9 , it is possible to derive the unsteady aerofoil hysteresis loops for $C_{l-} \alpha$ and $C_{d p^{-}}$ $\alpha$. Figs. 10(a)-(f) illustrate the hysteresis loops for the different wind speeds and radial locations. The 2D steady aerofoil data obtained from the Delft wind tunnel experiments are also shown. The 3D steady aerofoil data, which were derived using the blade pressure measurements for non-yawed conditions together with the free-wake code are also included. The hysteresis plots are helpful in establishing whether any particular blade section is operating in an attached or stalled-flow regime. One should keep in mind that in a yawed rotor, these loops are not only due to cyclic angle of attack variations, but also due to a cyclic variation of the flow velocity relative to the blades.

At $\mathrm{U}=5 \mathrm{~m} / \mathrm{s}$ (Figs. 10(a) and (b)), the angles of attack are small at all radial locations. Consequently the flow over the blades is expected to be fully attached throughout the whole blade revolution. Both the lift and drag hysteresis loops are considerably wide at inboard section $(\mathrm{r} / \mathrm{R}=0.3,0.47)$ which implies that unsteady effects here are significant even though no stall is present. At the other radial locations $(\mathrm{r} / \mathrm{R}=0.63,0.8$ and 0.95$)$, the loops are very narrow and thus unsteady effects here are negligible. At the higher windspeeds ( $\mathrm{U}=10$ and $15 \mathrm{~m} / \mathrm{s})$, the angle of attack goes beyond the 3D steady stalling angle this results in dynamic stall. It may be observed that much larger phase variations in $C_{l}$ and $C_{d p}$ are present than for attached flow conditions $(\mathrm{U}=5 \mathrm{~m} / \mathrm{s})$. This is a result of significant hysteresis in the flow structures. The values of $C_{l}$ and $C_{d p}$ at the same angle of attack will vary on whether the flow is separating or reattaching. In some severe cases however, flow re-attachment may not take place since the minimum angle of attack will be too high. Figs. 10(c)-(f) indicate that dynamic stall is most severe at the inboard blade sections. One may observe that the unsteady lift and drag coefficients may well exceed not only the 2D steady values, but also the 3D steady values derived for non-yawed conditions.

Some important comments concerning the derived hysteresis loops are:

- at each wind speed the hysteresis loops for both $C_{l}$ and $C_{d p}$ are larger at the inboard blade sections and unsteady effects are less pronounced at the outboard regions. However severe unsteady effects may still take place at the blade tip, especially at high angles of attack (refer to Fig. 10(e) for $r / R=0.95$ ) 
- in general, both the $C_{l}$ and $C_{d p}$ hysteresis loops are closer to the 3D steady values than to the corresponding 2D steady data. This is observed at both attached and stall conditions and it implies that $3 \mathrm{D}$ effects have a significant role in unsteady flow behavior of wind turbine blades, in particular stall-delay. This implies that a correction for 3D effects is also necessary when correcting 2D static aerofoil data for unsteady aerodynamic conditions.

In each of these hysteresis plots, the reduced frequency $k$ is included. $k$ is a very important parameter that is used to characterize the unsteadiness at which an aerofoil is operating. The reduced frequency is given by the equation:

$$
k=\frac{\Omega c}{2 V_{r}}
$$

When $k=0$, the flow is steady. When $0<k<0.05$, the unsteadiness in the flow is minimal and the flow may be assumed to be quasi-steady. Higher values of $k$ are considered unsteady. Values of $k$ equal to 0.2 and above are considered highly unsteady. Figures 10 show that the obtained values for $k$ at the different radial locations and wind speeds of the NREL rotor. Since in a yawed rotor the relative velocity of the flow at each blade section $\left(V_{r}\right)$ changes with blade azimuth angle $(\phi)$, the maximum and minimum values are included. The maximum and minimum values of $k$ occur at approximate blade azimuth angles of 0 and 180 deg respectively. It may be observed that the highest reduced frequencies occur at low $r / R$ values. However, changing the windspeed does not alter significantly the reduced frequencies at each radial position. This is a consequence of the fact that for the operating conditions of this rotor, the tunnel speed was much smaller than the rotor angular speed $(U<<\Omega r)$. Therefore the changes only had very small influences on $V_{r}$.

More detailed analysis of the unsteady aerodynamic effects may be carried out by analyzing these derived hysteresis loops in conjunction with the analysis of the unsteady chordwise blade pressure distributions measured in the wind tunnel. The latter analysis is very helpful in identifying the flow characteristics on the blades at each angle of attack, as described by Schreck et al. ${ }^{16,21}$.

\section{Bound Circulation Distributions at Blades}

Figure 11 illustrates 3D plots of the bound circulation distributions at the blades as a function of radial position $(r / R)$ and blade azimuth angle $(\phi)$. For lowest windspeed $(\mathrm{U}=5 \mathrm{~m} / \mathrm{s})$, at which the flow over the blades is considerably attached, the 3D plot takes the form of a saddle. At each blade azimuth angle, the peak bound circulation occurs close to the middle blade section. Also, the peak circulation is highest at blade position equal to $0 / 360 \mathrm{deg}$ and is lowest at about $180 \mathrm{deg}$. At high wind speeds, where separated flow conditions are known to occur $(\mathrm{U}=10,15 \mathrm{~m} / \mathrm{s})$, the saddle-shaped distribution is no longer present and a rather irregular distribution occurs. The peak bound circulation tends to shift towards the blade root region of the blade, where in fact the highest lift coefficients are noted. However the peak bound circulation is still observed close to blade position $0 / 360$.

\section{Wake Plots}

Figures 12 and 13 present the free-wake plots derived from the measured aerodynamic loading on the blades at $\mathrm{U}$ $=5$ and $15 \mathrm{~m} / \mathrm{s}$. Color coding is used to denote the trailing and shed circulation in the near wake. For each windspeed, two plots are presented: one for trailing circulation, the other for shed circulation. The color coding is very helpful in better understanding how the unsteady bound circulation at the blades (refer to Fig. 11) eventually diffuses into the wake downstream of the rotor in the form of trailing and shed circulation. The presence of roll-up of the vortex sheets is apparent. Since in a yawed rotor, the bound circulation is a function of rotor azimuth angle, the wake trailing and shed circulation will be time dependent. Looking at Figs. 12 and 13 will reveal that, at the lowest windspeed $(\mathrm{U}=5 \mathrm{~m} / \mathrm{s})$, the trailing circulation in the wake tends to be concentrated towards the blade tip and root regions. This is a result of the fact that the bound circulation at the blades is highest at the middle sections, but then decreases steadily to zero towards the blade tip and root (refer to Fig. 11). The trailing circulation at the tip region is positive, while it is negative at the root region. For high windspeed conditions however, the bound circulation distribution is irregular and this yields considerable levels of 'horse-shoe' trailing circulation to be released from the middle blade sections. This is especially observed at $U=15 \mathrm{~m} / \mathrm{s}$ (Fig. 13(c)). Consequently, the trailing circulation is more dispersed though the wake.

The shed circulation levels are small at $U=5 \mathrm{~m} / \mathrm{s}$ when compared with the trailing circulation levels. At higher windspeeds $(\mathrm{U}=10,15 \mathrm{~m} / \mathrm{s})$, higher shed circulation levels are observed at local spots of the wake vortex sheets. However the shed circulation across most of the vortex sheets remains small in magnitude in comparison with the trailing circulation. 

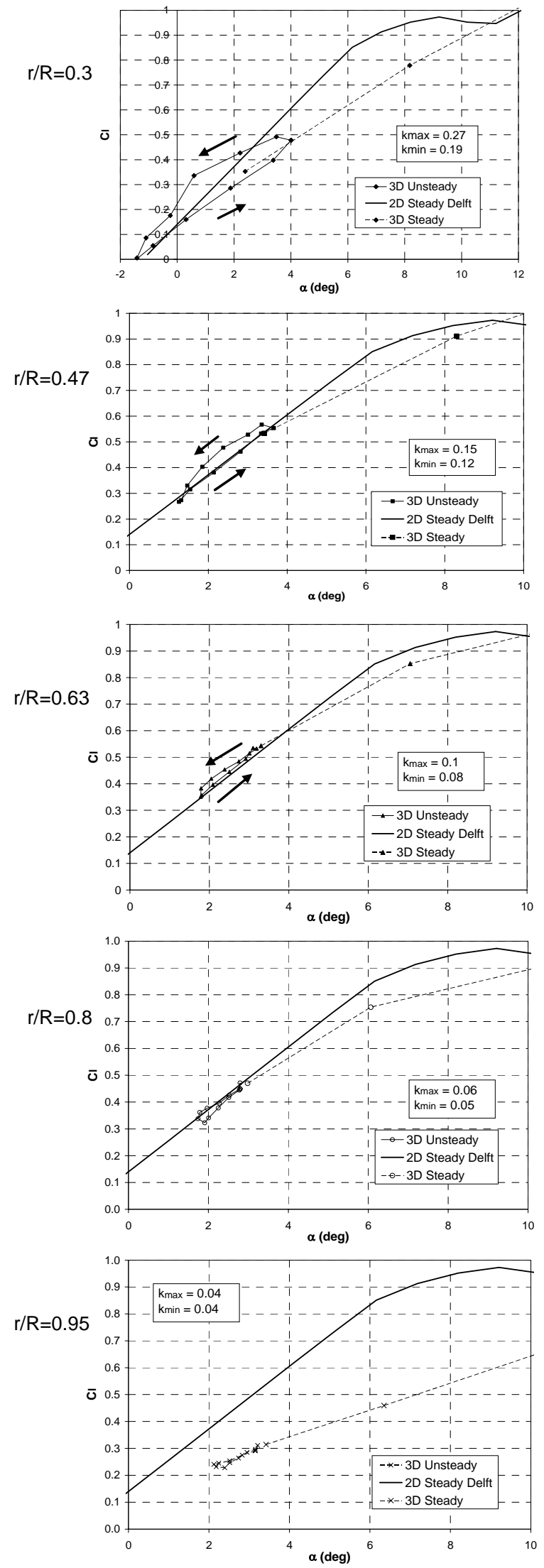

Fig. 10(a): $U=5 \mathrm{~m} / \mathrm{s}$
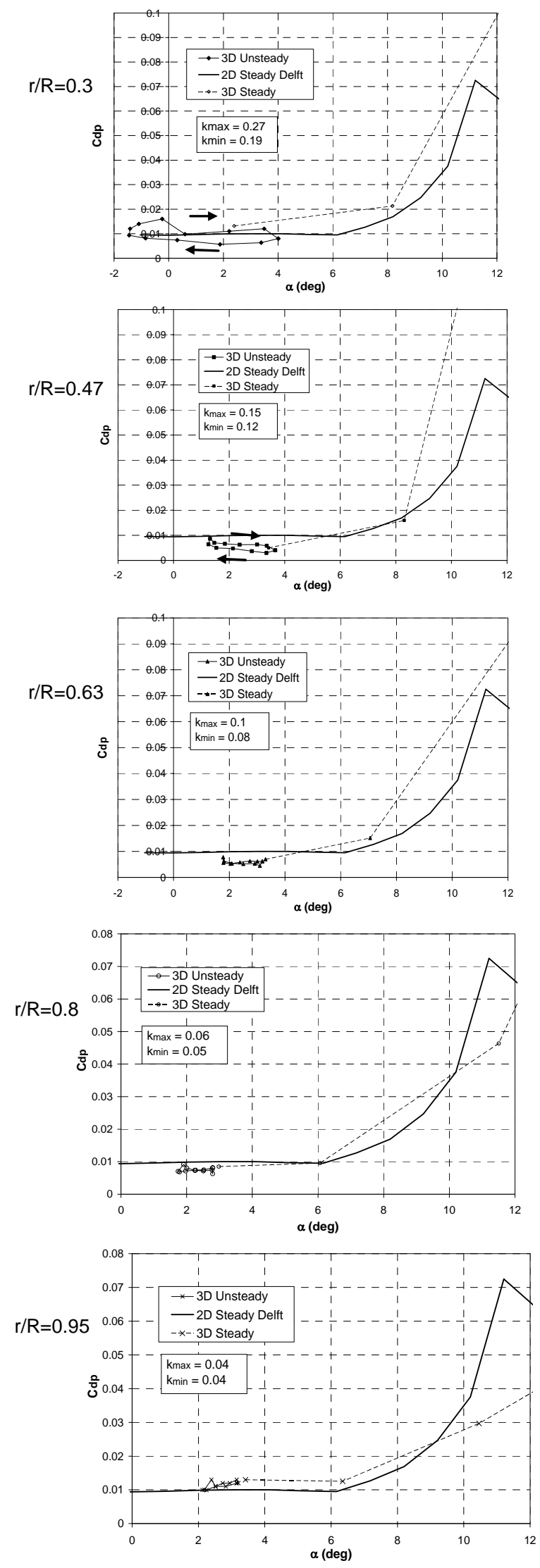

Fig. 10(b): $U=5 \mathrm{~m} / \mathrm{s}$ 

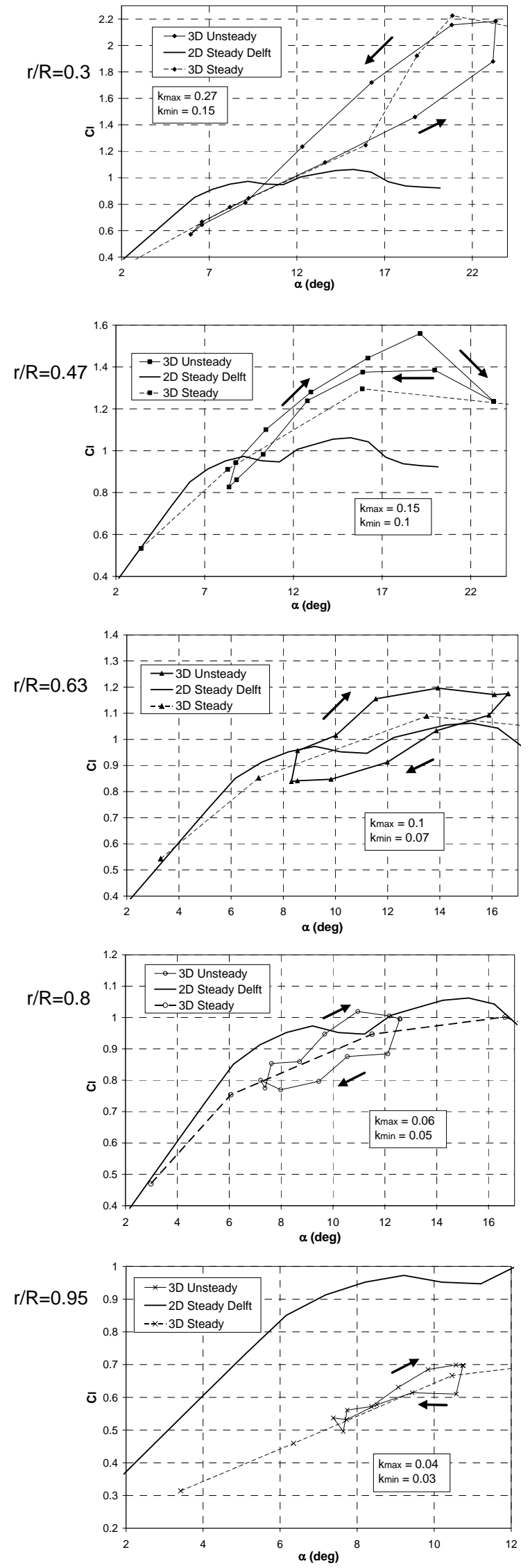

Fig. 10(c): $U=10 \mathrm{~m} / \mathrm{s}$
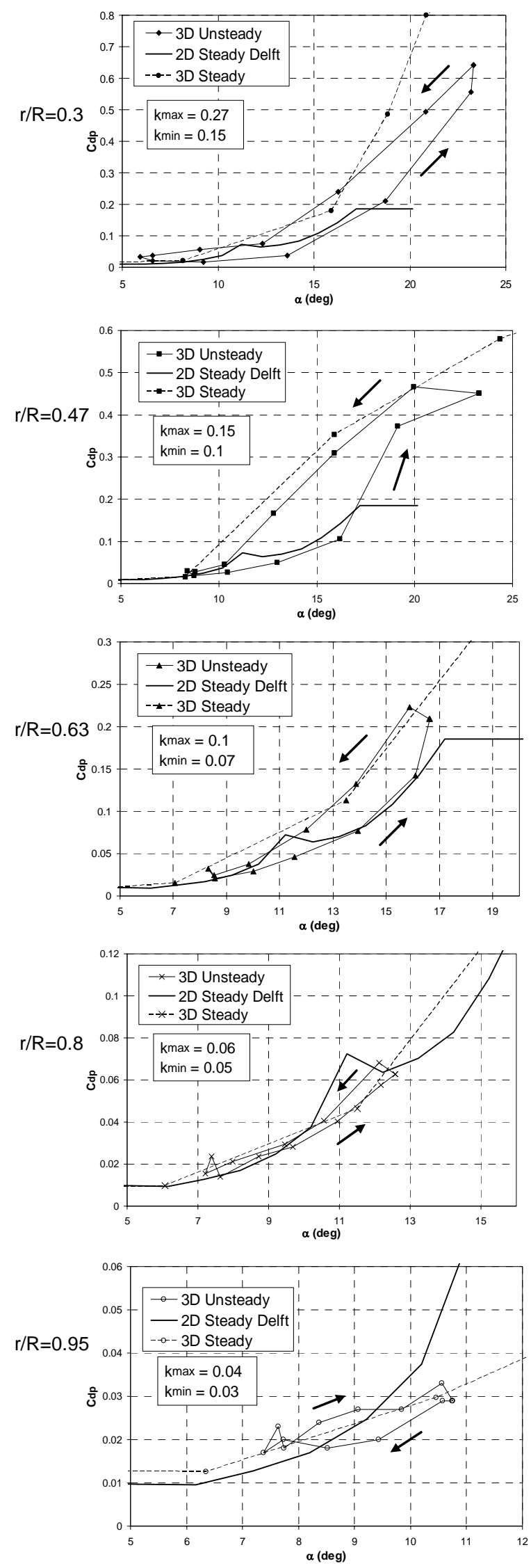

Fig. 10(d): $U=10 \mathrm{~m} / \mathrm{s}$ 

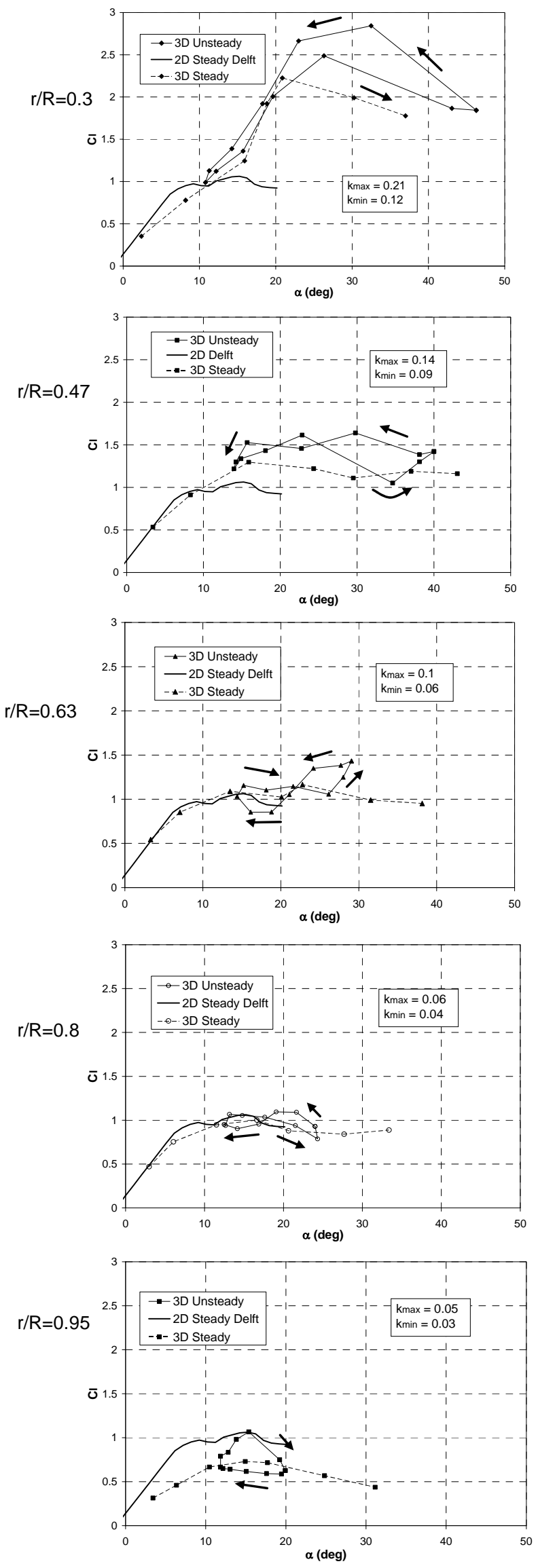

Fig. 10(e): $U=15 \mathrm{~m} / \mathrm{s}$
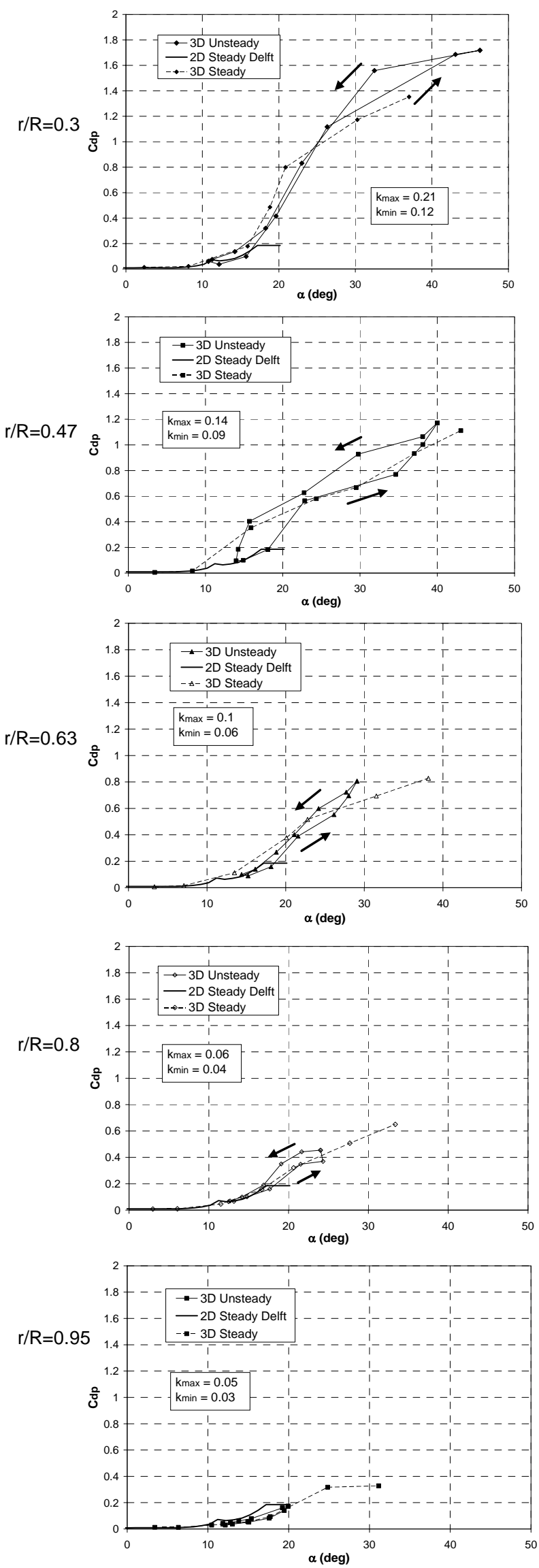

Fig. 10(f): $U=15 \mathrm{~m} / \mathrm{s}$ 


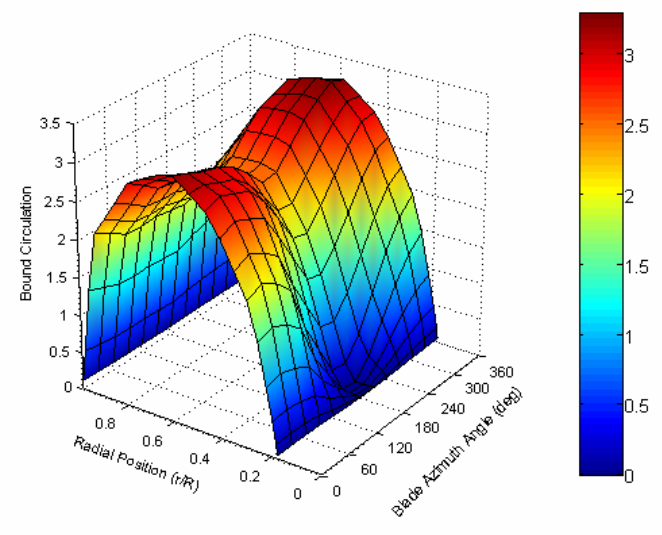

$U=5 \mathrm{~m} / \mathrm{s}$

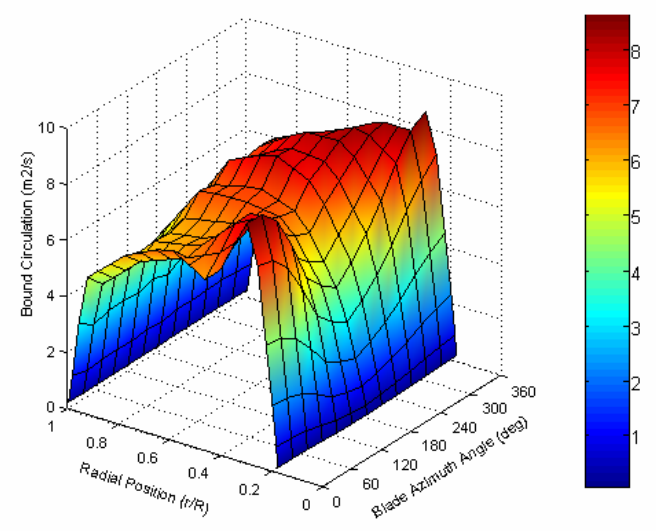

$U=10 \mathrm{~m} / \mathrm{s}$

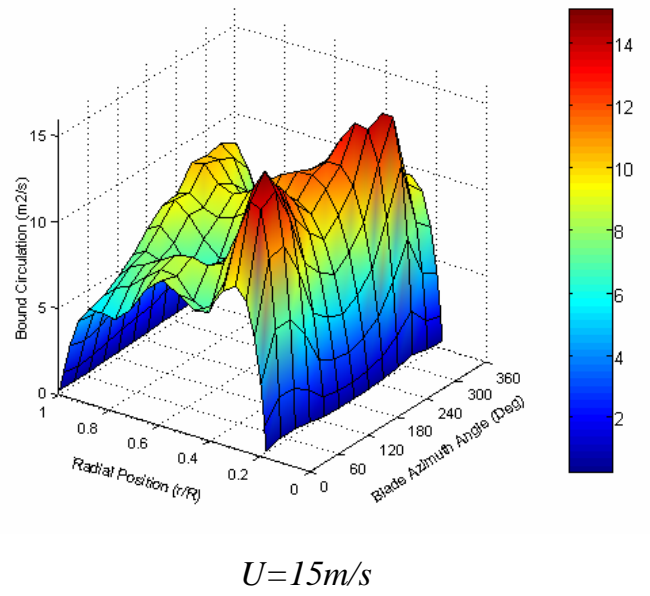

Figure 11: Variation of bound circulation at blades with radial location and blade azimuth angle

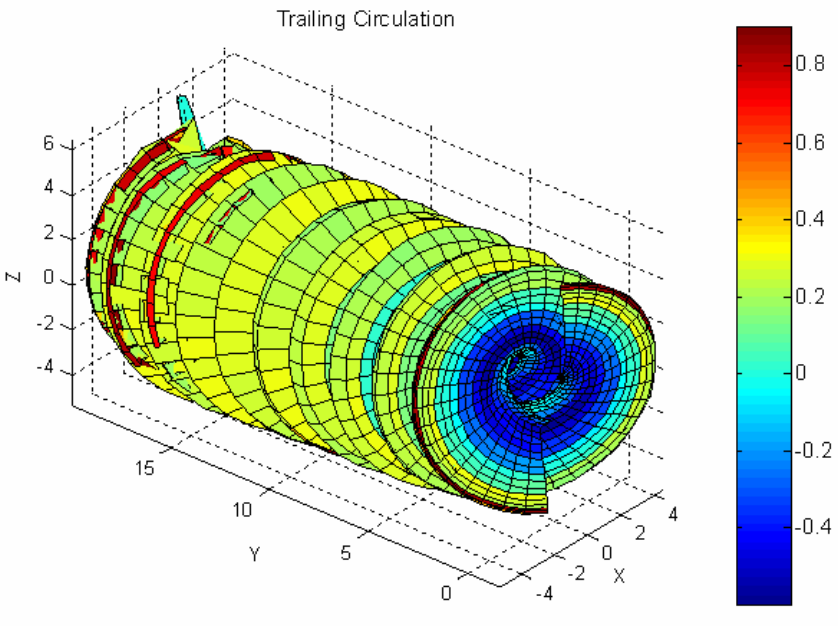

Fig. (a)

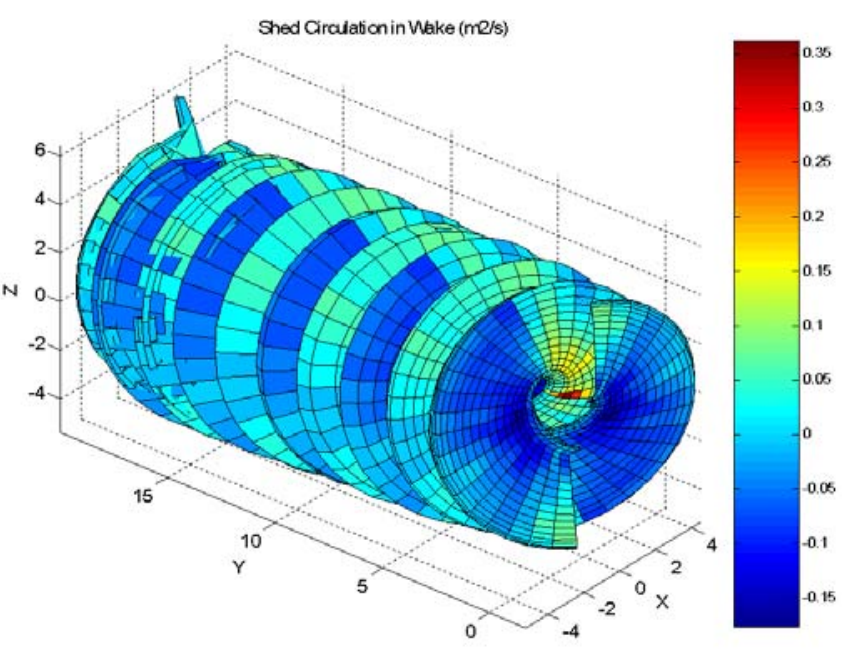

Fig. (b)

Fig. 12: Free-wake plots for $U=5 \mathrm{~m} / \mathrm{s}$. Color coding denotes trailing and shed circulation distribution in wake 


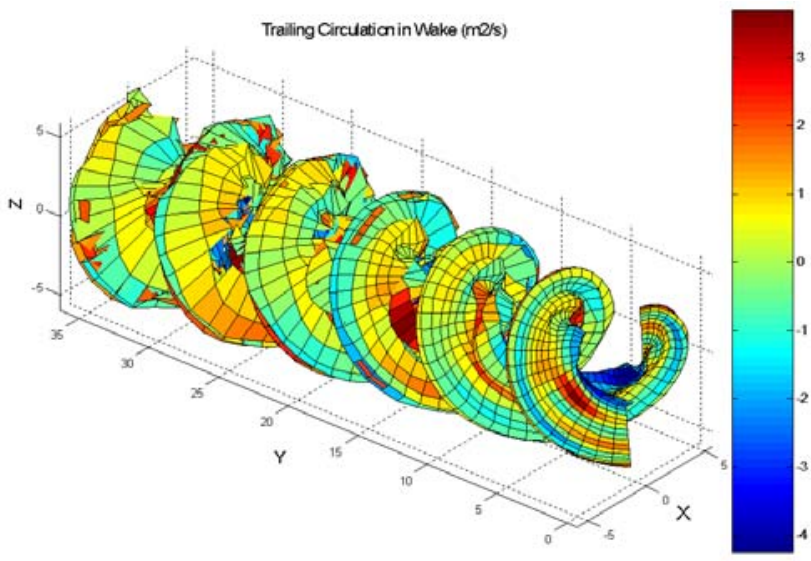

Fig. (c)

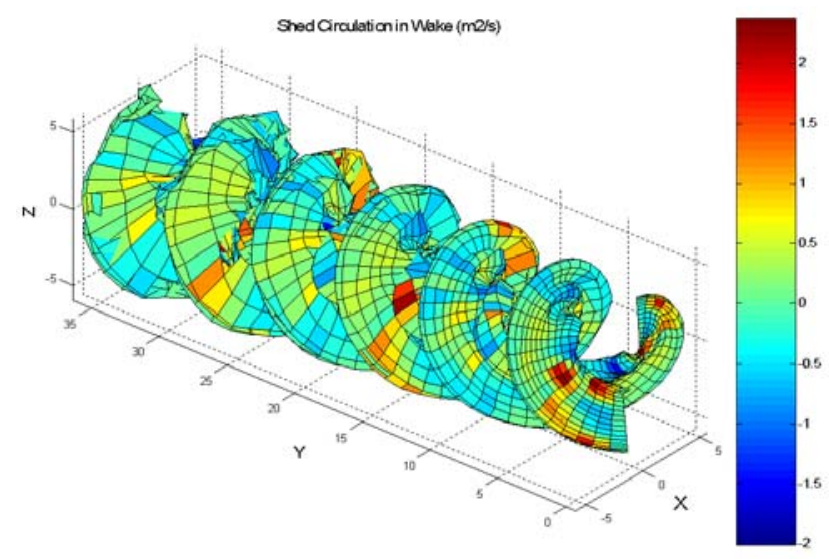

Fig. (d)

Fig. 13: Free-wake plots for $U=15 \mathrm{~m} / \mathrm{s}$. Color coding denotes trailing and shed circulation distribution in wake

\section{E. Induced Velocities at Rotorplane}

Figures 14 illustrate the unsteady axial induction factors at the rotorplane as predicted by the free-wake plot in accordance with the blade pressure measurements taken in the wind tunnel. Figs. 14 (a)-(c) show the variation of the azimuthally-averaged axial induction factor (i.e. annular averaged axial induction factor, $a_{1}$ ) at the rotorplane with rotor azimuth angle for different radial locations. Figs. 14 (d)-(f) show the variation of the axial induction factor at the blade lifting line $\left(a_{1, c}\right)$ with blade azimuth angle for different radial locations. The corresponding tangential and radial induction factors were also calculated but these are not included in this paper as they were found to be very small compared to the rotor angular speed.

When the windspeed is low $(\mathrm{U}=5 \mathrm{~m} / \mathrm{s})$, the free wake model predicts an azimuthally-averaged axial induction factor that is almost constant with rotor azimuth angle. Higher the windspeeds increase the cyclic variation of $a_{1}$. The cyclic component is highest at the inboard blade sections. The cyclic variation has a frequency equal to $2 p$ and this is because the rotor has two blades.

The variation of the axial induced velocity at the blade lifting line with blade azimuth angle is nearly sinusoidal at low wind speeds $(\mathrm{U}=5 \mathrm{~m} / \mathrm{s})$, especially at the outboard regions of the blades (see Fig. 14 (d)) but then becomes very irregular at higher windspeeds ( $\mathrm{U}=10,15 \mathrm{~m} / \mathrm{s}$; see Figs. 14 (e),(f)).

At low wind speeds, the sinusoidal variation $a_{1, c}$ with $\phi$ has a phase angle that changes depending on the radial position $(r / R)$ of the blade section. This is due to the fact that in a skewed wake, the proximity of the blades to the wake circulation is a function of both blade azimuth angle and radial location. Recall from section V D. that at the low windspeeds, the trailing circulation in the wake tends to be concentrated at the tip and root regions. The outboard blade sections are in general closer to the wake circulation that is originating from the blade tips at blade azimuth position of 90 degrees than at $270 \mathrm{deg}$. Consequently the induced velocity at the outboard blade sections is higher at $90 \mathrm{deg}$ than at $270 \mathrm{deg}$, as in fact depicted in Fig. 14(d). As one moves inboard, the proximity of the blade sections to the wake circulation originating from the tip decreases, but this is followed by an increased proximity to the wake circulation originating from the blade roots. This causes the maximum induced velocity to occur at a different blade azimuth angle, thus changing the phase angle of the variation of $a_{1, c}$ with $\phi$.

At higher windspeeds $(\mathrm{U}=10$ and $15 \mathrm{~m} / \mathrm{s})$, the presence of 'horse-shoe' trailing circulation levels at the middle blade sections remove the sinusoidal variation of $a_{1, c}$ with $\phi$ at all radial locations, making it very irregular.

From the results of this study, one can easily remark the following deficiencies of Glauert's equation (1) (or similar models):

- Glauert's model assumes that the azimuthally-averaged axial induced velocity $\left(a_{1}\right)$ does not vary with rotor azimuth angle. Although this assumption may be realistic at high tip speed ratios, it does not necessarily apply for high windspeed conditions where the flow is separated. 
- Glauert's model does not cater for the phase angle change with radial location of the variation $a_{1, c}$ with $\phi$, resulting from root circulation. Also as noted in Ref. 10, root circulation effects may create a induced velocity distribution that has a higher harmonic content than only $1 \mathrm{p}$.

- Glauert's model is invalid for low tip speed ratios with stalled flows were the variation $a_{1, c}$ with $\phi$ becomes very irregular.

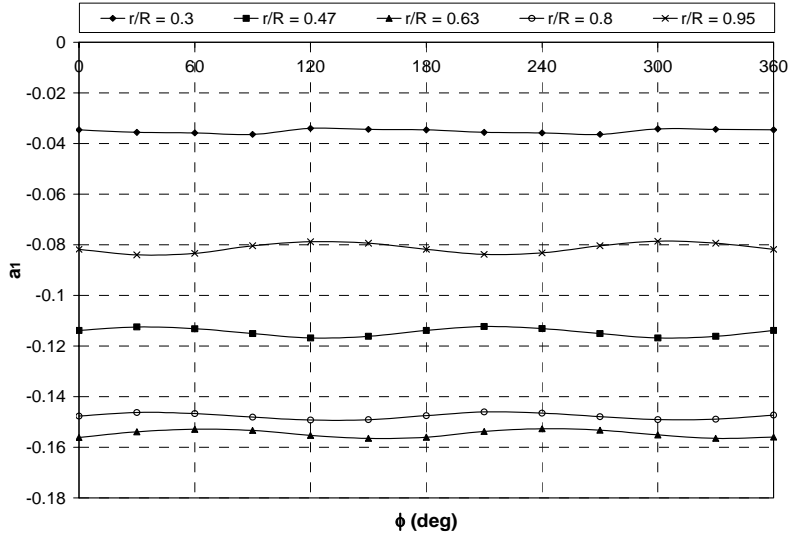

Fig. 14(a): $U=5 \mathrm{~m} / \mathrm{s}$

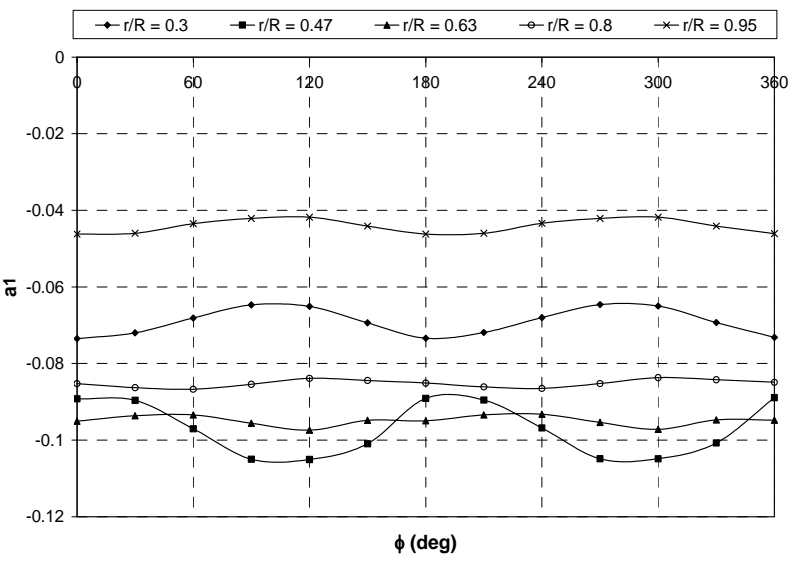

Fig. 14(b): $U=10 \mathrm{~m} / \mathrm{s}$

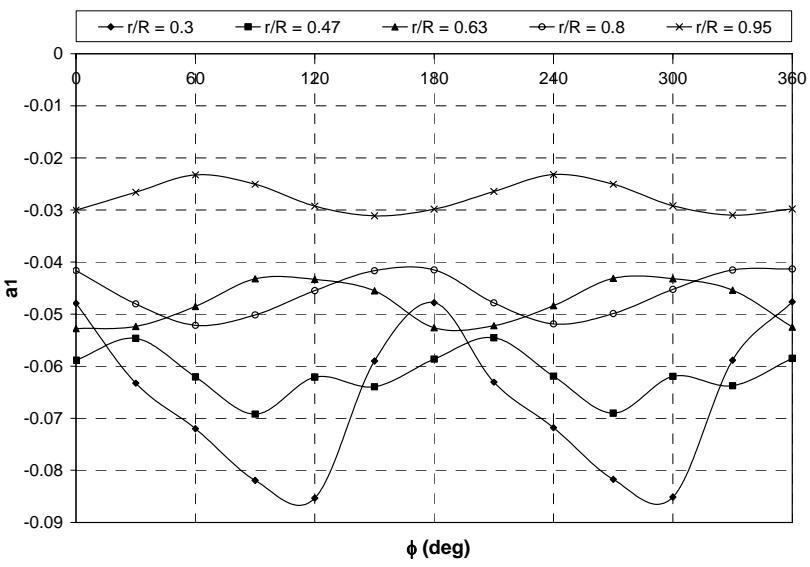

Fig. 14(c): $U=15 \mathrm{~m} / \mathrm{s}$

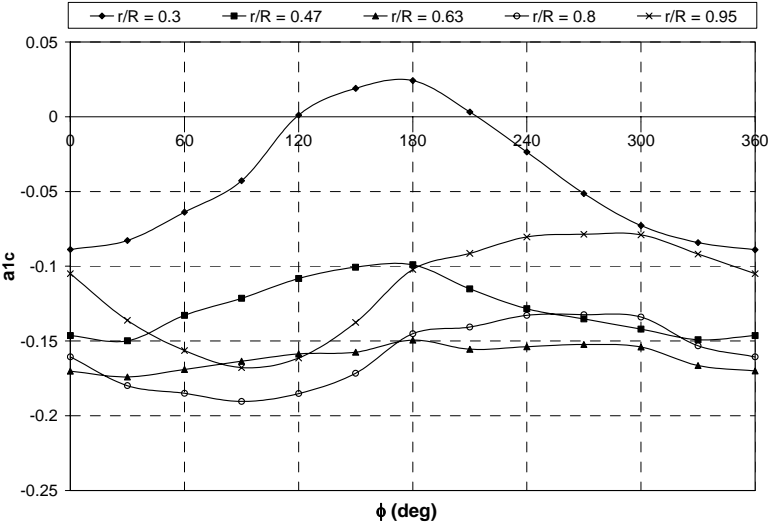

Fig. 14(d): $U=5 \mathrm{~m} / \mathrm{s}$

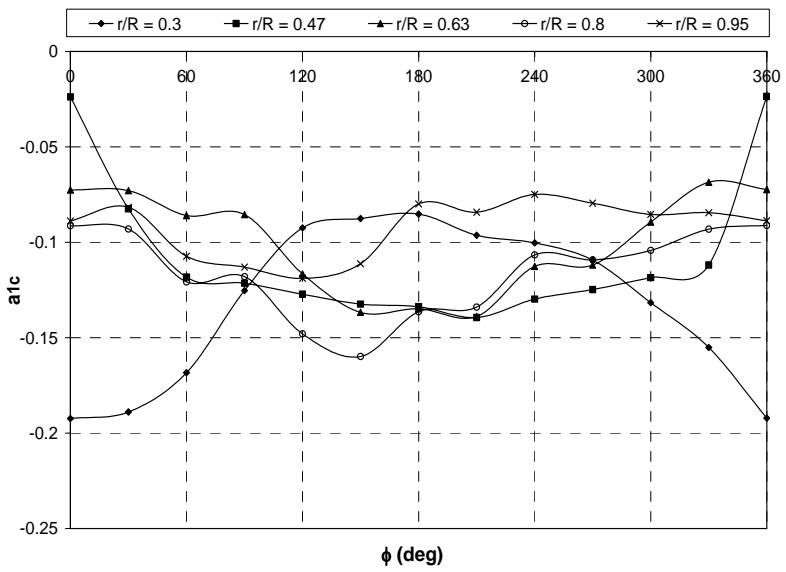

Fig. 14(e): $U=10 \mathrm{~m} / \mathrm{s}$

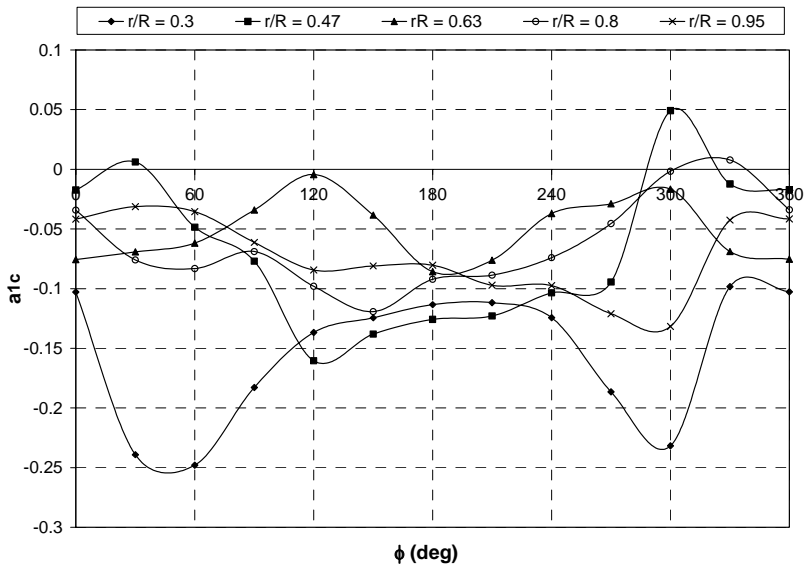

Fig. 14(f): $U=15 \mathrm{~m} / \mathrm{s}$ 
As already described in section III, in the free vortex computations of this study, the induction at the lifting line of the blades is due to the free-wake trailing and shed circulation and due to the trailing circulation of the prescribed far wake model. The induction at the lifting line due to bound circulation from the two blades is zero. This is a consequence of the fact that a lifting line model is used to represent the blades and also because both lifting lines are in-line with one another (no blade coning). As already mentioned in section IV D., in the free-wake calculations, the near wake parameter $n R e v$ was selected large enough so that the induction contributed by the far wake is very small. Thus it may be assumed that the total induction is due to trailing and shed circulation of the near wake only. Using this free-wake model, it is possible to calculate the induced velocity components resulting from the near wake trailing and shed circulation separately. In this way it would be possible to determine which circulation type has the greater influence on the flow at the blades. The induced velocities of Fig. 14 are shown again in Figs. 15 for U=5 and $15 \mathrm{~m} / \mathrm{s}$, but including the individual induced velocity components due to trailing and shed circulation. The induced velocity by the shed circulation in general very small. This is observed even at $U=10 \mathrm{~m} / \mathrm{s}$. This proves that the trailing circulation is by far more dominant than the shed counterpart. The induction component due to the far wake alone is also included in Fig. 14 and as it may be observed it is very small compared to the total induction.

\section{F. Aerodynamic loads}

The unsteady lift and drag coefficients and induced velocities derived by the free-wake vortex model were used to calculate the low-speed shaft torque and the blade flap/edgewise bending moments using the blade-element theory equations. These results are shown in Figs. 16 (a)-(c) were they are noted by plots 'Free-wake'. As a cross-check for these calculations, the same loads were computed directly from the experimental data by using a linear interpolation to obtain a distribution for $C_{n}, C_{t}$ and $\mathrm{Q}_{\mathrm{NORM}}$ across the whole blade span and using the following equations:

$$
\begin{aligned}
& L S S T Q_{\tau}=\int_{b=0}^{b=B-1} \int_{R_{n u b}}^{R_{i p}} \operatorname{QNORM}_{\tau_{b, i}} \times\left(C_{t_{\tau_{b, i}}} * \cos \left(\theta_{i}\right)+C_{n_{\tau_{b, i}}} * \sin \left(\theta_{i}\right)\right) \times r_{i} \times d r \\
& R F M_{\tau}=\int_{R_{\text {mb }}}^{R_{\text {ip }}} \operatorname{QNORM}_{\tau_{b, i}} \times\left(C_{n_{\tau_{b, i}}} * \cos \left(\theta_{i}-\theta_{\text {tip }}\right)-C_{t_{\tau_{b, i}}} * \sin \left(\theta_{i}-\theta_{\text {tip }}\right)\right) \times\left(r_{i}-r_{\text {strain gauge }}\right) \times d r \\
& R E M_{\tau}=\int_{R_{n, b}}^{R_{i p}} \operatorname{QNORM}_{\tau_{b, i}} \times\left(C_{n_{\tau_{b, i}}} * \cos \left(\theta_{i}-\theta_{\text {tip }}\right)+C_{t_{\tau_{b, i}}} * \sin \left(\theta_{i}-\theta_{\text {tip }}\right)\right) \times\left(r_{i}-r_{\text {strain gauge }}\right) \times d r
\end{aligned}
$$

where $r_{\text {strain gauge }}$ is the radial location of the strain gauges measuring the blade root flap/edge moments. $\theta_{\text {tip }}$ is included in the above equations because the strain gauges installed at one of the rotor blades were oriented such that they measure the flapping moment about an axis parallel to the chordline of the blade tip section (Ref. 28). In the linear interpolation, $C_{n}$ and $C_{t}$ at the blade tips were set equal to zero. The trapezium rule was adopted for integrating numerically across the whole blade span. These results are included in Figs. 16 and are referred to 'Exp'. The loads measured directly using strain-gauges are also included in Figs. 16. The results calculated by the freewake model and those derived directly from the pressure measurements ('Exp') agree very well. However agreement with the loads measured by the strain gauges is not as good. The reasons for the discrepancies between the free-wake results and the strain gauge measurements are various. First of all, the free-wake model only calculates aerodynamic loads and the loads due to gravity and structural dynamics are not accounted for. Gravitational loads are not included and thus the computed edgewise moment does not include the cyclic component induced by the blade weight. This leads to a large discrepancy in Figs. 16(c). In the free-wake model, the rotor blades are assumed to be rigid and the influence of blade deflection on the aerodynamics of the blades are excluded in the modeling. Also aerodynamic influences due to the tower and rotor nacelle were not included. However the very good agreement obtained between the loads calculated by the free-wake code ('Free-wake') and those derived directly from the pressure measurements ('Exp') might imply that these aerodynamics influences are minimal.

\section{F. Comparison of Inflow Angle}

Figs. 17 compare the unsteady local flow angle (LFA) variations calculated by the free-wake model with those measured by the flow direction probes at $\mathrm{U}=10 \mathrm{~m} / \mathrm{s}$. The angle of attack is also included. Ideally the probes and freewake model LFA results should be the same but this is not the case due to various sources of error. The maximum discrepancy is about 5 degrees and occurs at the inboard section $r / R=0.34$ (refer Figs. 17). To assess the uncertainty due to arbitrary choice of the viscous parameters, the LFA was computed using the free-wake model at different values of $\left(\delta_{v}, S_{c}\right)$ : at $(10,0.1),(100,1)$ and $(500,1)$ : The maximum deviation in the LFA due to different $\left(\delta_{v}, S_{c}\right)$ values 
was found to be only $0.54^{0}$ at $r / R=0.91$. Sources of error in the free-wake modeling are due to the fact that the blades are modeled as a lifting lines only and also because blade deflection effects are not accounted for. However an apparent source of error is due to the flow direction measuring equipment itself. One should also keep in mind that the probe was subjected to highly unsteady flow situations. At $\mathrm{U}=10 \mathrm{~m} / \mathrm{s}$, the free-wake model estimates that the time rate of change of angle of attack reaches a peak of about $\pm 78 \mathrm{deg} / \mathrm{s}$ (refer to Fig. 9(b)) at the inboard sections. Further work is required to establish the accuracy with which five-hole pressure probes manage to measure the LFA at such high levels of unsteadiness.

\section{Conclusion}

This paper describes a method in which a free-wake vortex model is used in conjunction with the blade pressure measurements to investigate the aerodynamic behavior of a wind turbine in yaw. It is shown that, using a free-wake vortex model, it is possible to derive the unsteady angle of attack distributions from knowledge of the aerodynamic normal and tangential forces acting on the blades. These distributions are used to estimate the 3D unsteady lift and drag coefficients. The unsteady 3D aerofoil lift and drag hysteresis loops are found to vary significantly both with radial position and windspeed. Also, the unsteady $3 \mathrm{D}$ aerofoil coefficients may exceed the corresponding 3D values derived for non-yawed conditions. As regards the variation of the axial induced velocity at the blades with blade azimuth angle, it is observed that this variation is only sinusoidal for low wind speeds and outboard blade stations. The phase angle of this variation changes with radial position. At high windspeeds very close to (or in) stall, this variation becomes very irregular and this may impose difficulties in developing improved simple engineering models to be incorporated into BEM-based aeroelastic design codes. One should recall the fact that unlike helicopters, wind turbines need to be operated very close or in the stall regime to be able to maximize power extraction.

The free-wake plots are plotted together with the corresponding trailing and shed circulation distributions. These provide a pictorial explanation of how the time-dependent bound circulation formed at the blades is eventually diffused into the wake under the action of complex 3D flows. Trailing circulation levels are found to be in general much more dominant than shed circulation levels at all windspeeds. This is mainly due that the fact that the yaw angle is small. It is expected that for larger yaw angles (above 30deg), the shed circulation levels are become higher.

The maximum discrepancy between the local inflow angles estimated by the free-wake vortex model and those measured by the probes was found to be about $5 \mathrm{deg}$. Further work is required to establish the accuracy with which five-hole probes measure the LFA in an unsteady and complex 3D flow field of a rotor in yaw.

\section{Future Work}

It is important to point out the fact that, although this study has successfully demonstrated the effectiveness of using a free-wake model in conjunction with blade pressure measurements to obtained further insight of rotor yaw aerodynamics, the results obtained are subject to the following uncertainties:

- The accuracy of the results is dependent on the accuracy with which the free-wake vortex model models the blades and wake. In this study, a lifting line representation of the blades was used and further work is required to investigate the accuracy of lifting-line models in representing the blades in yawed conditions. Lifting surface and panel methods are more accurate in capturing certain 3D flow effects around the blades. Further research is also required to validate the viscous modeling methodology adopted by the subject free-wake code.

- This study did not cater for the cycle-to-cycle variations observed in the measured values of $C_{n}$ and $C_{t}$. These variations were considerably high in yawed conditions (refer to Fig. 2). In this study, only the mean values of $C_{n}$ and $C_{t}$ were used in the calculations. Future work will include a stochastic analysis to account for such cycle-tocycle variations.

\section{Acknowledgments}

The authors would like to express their gratitude to Dr. Scott Schreck of the NREL for providing the data of the Phase VI wind tunnel experiments. The authors would also like to thank Ruud van Rooij for his discussion time during the course of this work. 

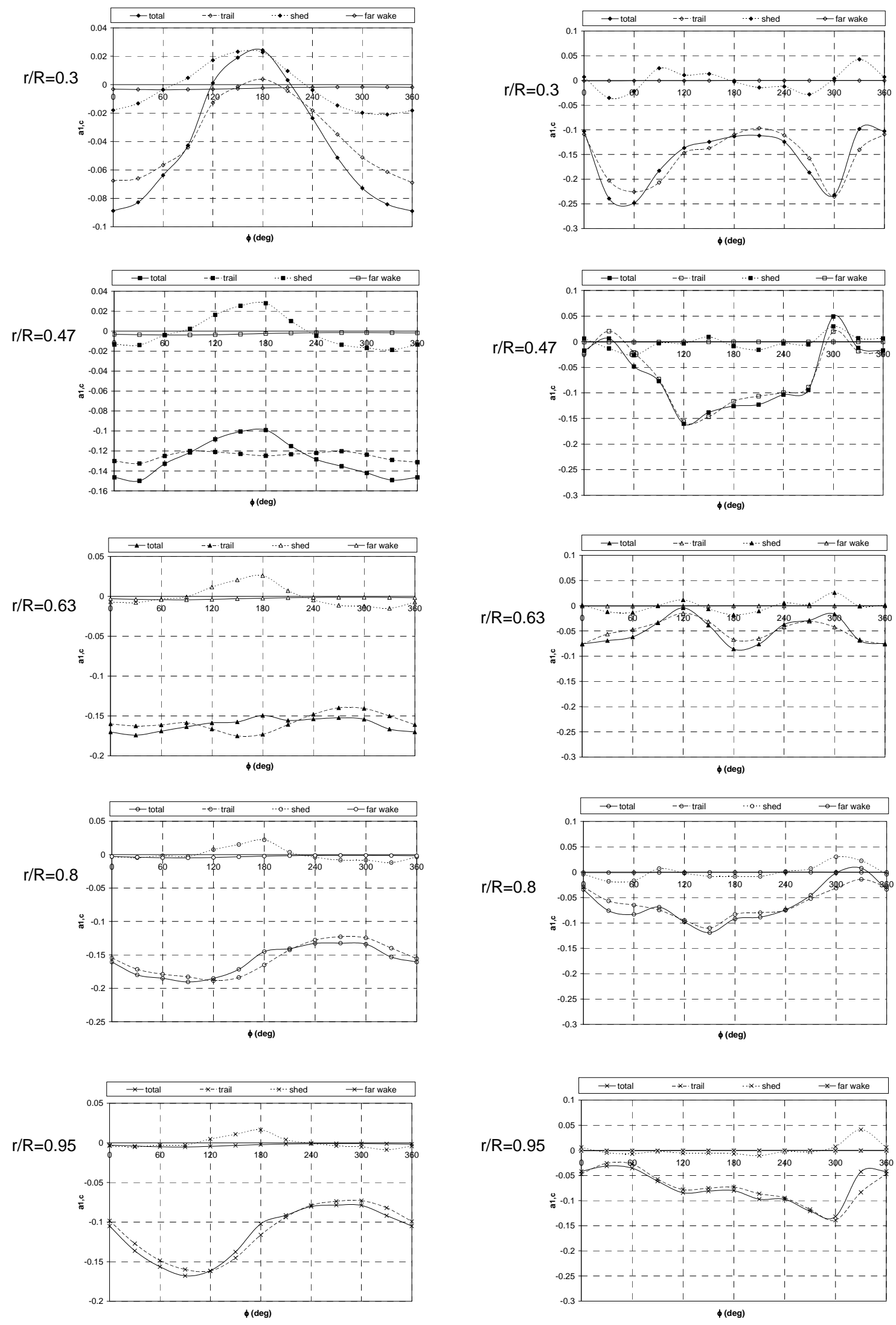

Fig. 15(a): $U=5 \mathrm{~m} / \mathrm{s}$

Fig.15(b): $U=15 \mathrm{~m} / \mathrm{s}$

American Institute of Aeronautics and Astronautics 


\section{Torque}
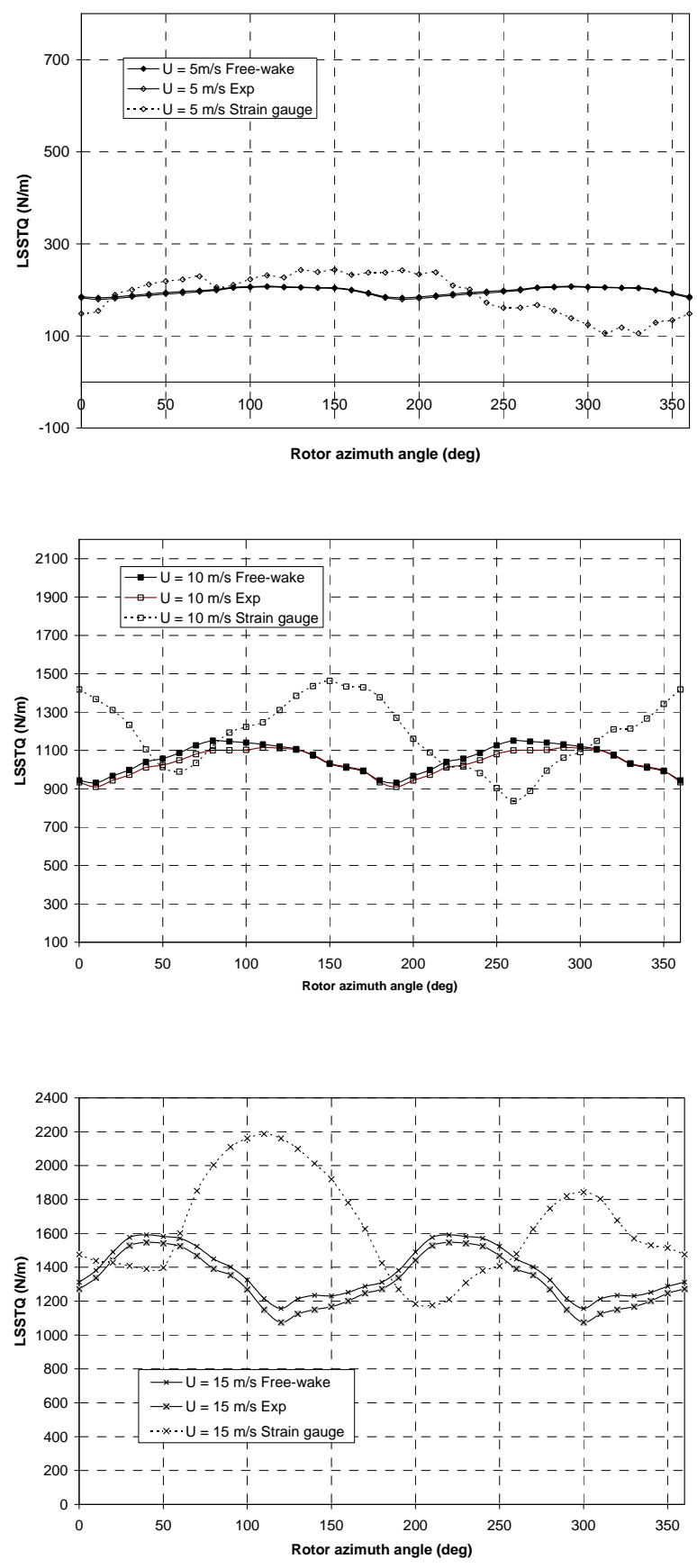

Fig. 16 (a)
Blade Root Flap Bending Moment
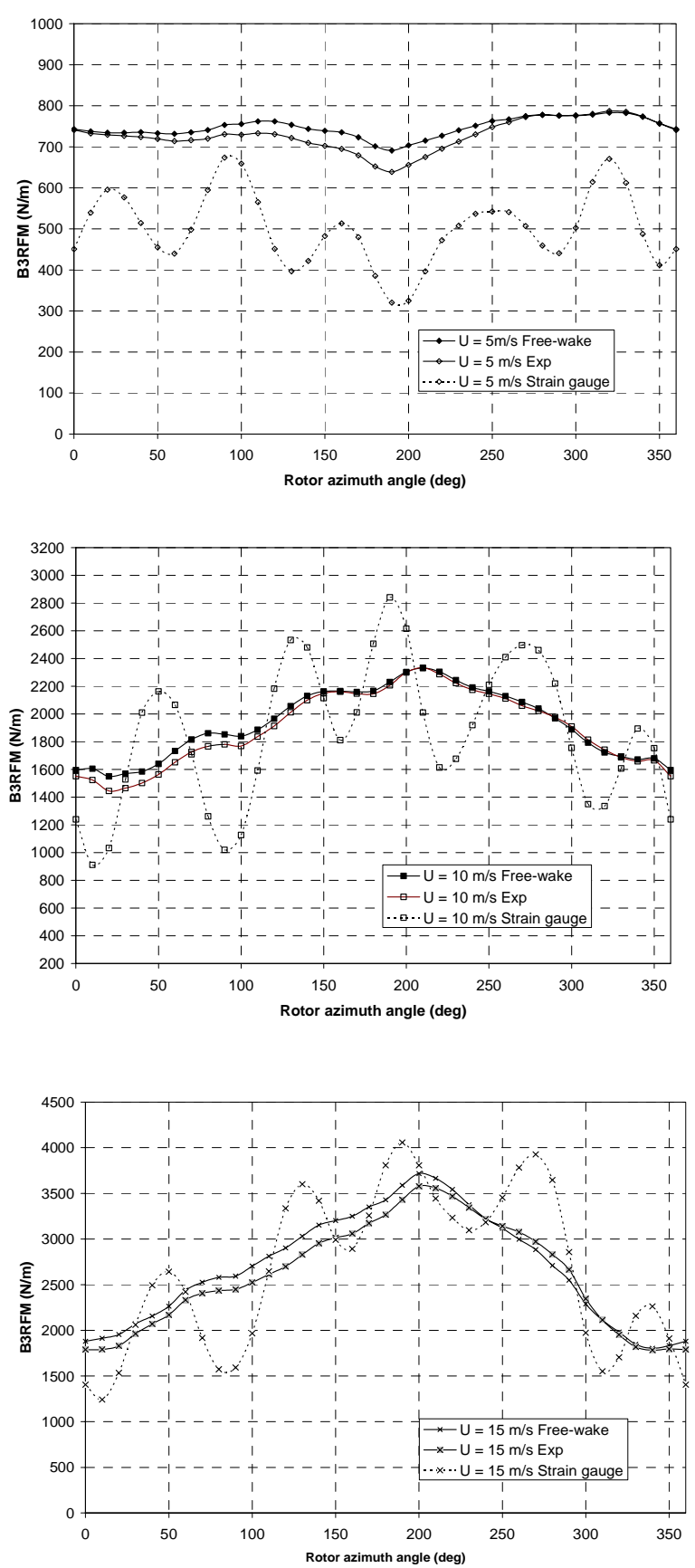

Fig. 16 (b) 
Blade Root Edgewise Bending Moment
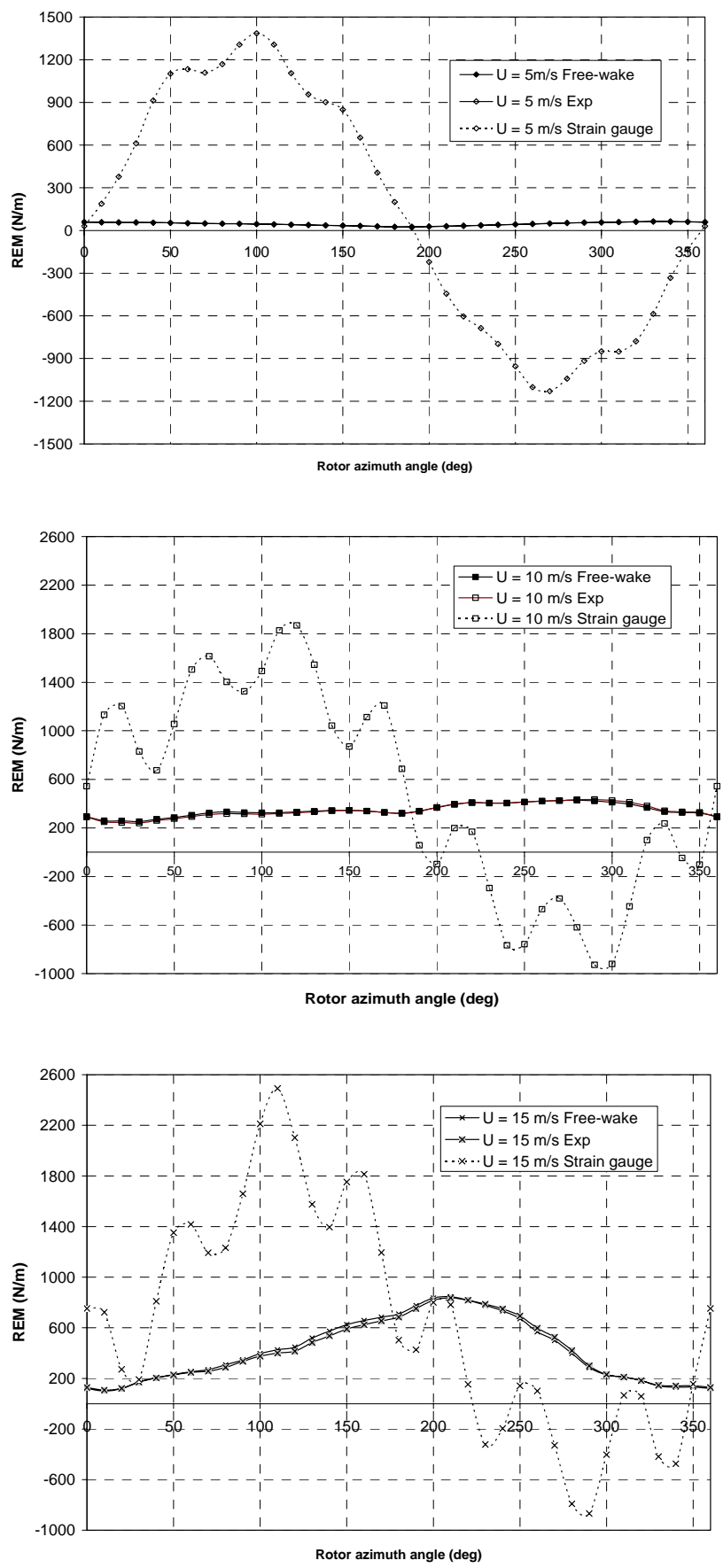

Fig. 16 (c)
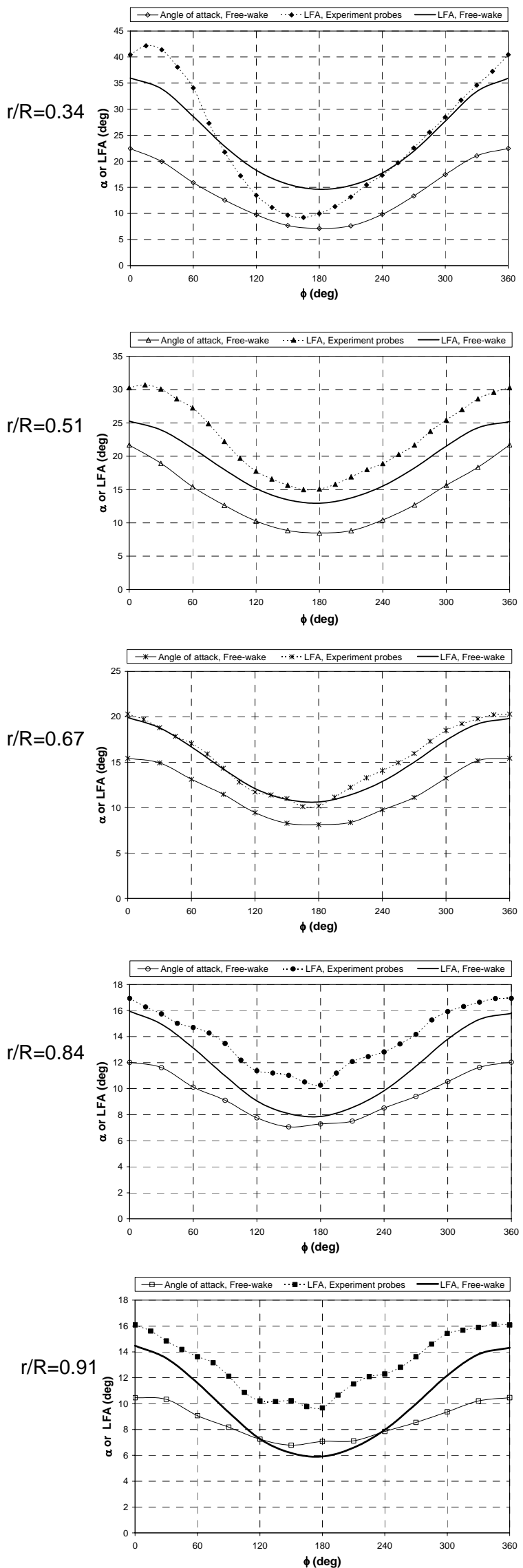

Fig. 17 


\section{References}

${ }^{1}$ Snel, H., Houwink, R., and Bosschers, J., "Sectional Prediction of Lift Coefficients on Rotating Wind Turbine Blades in Stall”, ECN-C-93-052, 1993.

${ }^{2}$ Du, Z. and Selig, M., “A 3D Stall-Delay Model for Horizontal Axis Wind Turbine Performance Predictions”, AIAA-98-0021, ASME Wind Energy Symposium, Reno, 1998.

${ }^{3}$ Chaviaropoulos, P.K. and Hansen, M.O.L., "Investigating Three-Dimensional and Rotational Effects on Wind Turbine Blades by means of a Quasi-3D Navier-Stokes Solver", Journal of Fluids Engineering, vol.122, June 2000, p.p. 330-336.

${ }^{4}$ Theodorsen, T., "General Theory of Aerodynamic Instability nd the Mechanism of Flutter," NACA Report 496, 1935.

${ }^{5}$ Leishman, J.G., "Principles of Helicopter Aerodynamics", Cambridge University Press, 2000.

${ }^{6}$ Leishman, J. G., "Challenges in Modelling the Unsteady Aerodynamics of Wind Turbines" Wind Energy, Vol. 5, No. 11, 2002, pp. 85-132.

${ }^{7}$ Leishman, J.G., "Modelling Sweep Effects on Dynamic Stall”, Journal of the Amer. Helicopter Soc., 34 (3), pp 18-29, 1989.

${ }^{8}$ Glauert, H., "A General Theory for the Autogiro", ARC R \& M 786, 1926.

${ }^{9}$ Vermeer, L.J., "Wind Tunnel Experiments on a Rotor Model in Yaw". Proceedings of the $12^{\text {th }}$ symposium on Aerodynamics of Wind Turbines, Lyngby, 1998, 11-20.

${ }^{10}$ Schepers J.G., "An Engineering Model for Yawed Conditions, Developed on the Basis of Wind Tunnel Measurements", AIAA-99-0039 ASME Conference, 1999, Reno, USA.

${ }^{11}$ Snel H. and Schepers, J.G. (ed), "Joint Investigation of Dynamic Inflow Effects and Implementation of an Engineering Method", Netherlands Energy Research Foundation, ECN-C-94-107, 1994.

${ }^{12}$ Snel H. and Schepers, J.G. (ed), "Dynamic Inflow: Yawed Conditions and Partial Span Pitch Control", Netherlands Energy Research Foundation, ECN-C-95-056, 1995.

${ }^{13}$ Schreck S., "The NREL Full-Scale Wind Tunnel Experiment - Introduction to the Special Issue", Wind Energy, Vol. 5, 2002, pp. 77-84.

${ }^{14}$ Simms, D., Schreck, S., Hand, M. and Fingersh, L.J., "NREL Unsteady Aerodynamics Experiment in the NASA-Ames Wind Tunnel: A Comparison of Predictions to Measurements," NREL Tech Report NREL/TP-500-29494, Golden, Colorado, June 2001.

${ }^{15}$ Tangler, J.L., "The Neboulous Art of Using Wind Tunnel Aerofoil Data for Predicting Rotor Performance", Wind Energy, Vol. 5, 2002, pp 245-257.

${ }^{16}$ Schreck, S. and Robinson, M., "Structures and Interactions Underlying Rotational Augmentation of Blade Aerodynamic Response", AIAA-2003-0520, Proceedings from 22nd ASME Wind Energy Symposium, Reno, 2002.

${ }^{17}$ Schmitz S., Chattot J.J., "Application of a 'Parallelized Coupled Navier-Stokes/Vortex-Panel Solver' to the NREL Phase VI Rotor", AIAA-2005-0593, Proceedings from 24th ASME Wind Energy Symposium, Reno, 2005.

${ }^{18}$ SØresen N.N, Michelsen J.A., Schreck S., "Navier-Stokes Predictions of the NREL Phase VI Rotor in the NASA $80 \mathrm{ft} X$ $120 \mathrm{ft}$ Wind Tunnel”, Wind Energy, Vol 5, 2002, pp. 151-169.

${ }^{19}$ Lindenburg, C., "Investigation into Rotor Aerodynamics - Analysis of the stationary measurements on the UAE phase VI rotor in the NASA-Ames wind tunnel”, ECN report ECN-C-03-025, 2003.

${ }^{20}$ Laino D.J., Hansen A.C and Minnema J.E., "Validation of the Aerodyn Subroutines Using NREL Unsteady Aerodynamics Experiment Data, Wind Energy, Vol 5, 2002, p.p. $227-244$.

${ }^{21}$ Schreck, S., Robinson, M, Hand, M. and Simms, D., "Blade Dynamic Stall Vortex Kinematics for a Horizontal Axis Wind Turbine in Yawed Conditions", ASME Journal of Solar Energy Engineering, Vol. 123, pp 272-281, Nov. 2001.

${ }^{22}$ Coton, F.N., Wang, T. and McD. Galraith, R. A., "An Examination of Key Aerodynamic Modelling Issues Raised by The NREL Blind Comparison," Wind Energy, 2002,; 5: 199-212.

${ }^{23}$ Tongchitpakdee C., Benjanirat S, Sankar L.N., "Numerical Simulation of the Aerodynamics of Horizontal Wind Turbines Under Yawed Flow Conditions", AIAA-2005-0773, Proceedings from 24th ASME Wind Energy Symposium, Reno, 2005.

${ }^{24}$ van Rooij R.P.O.M., Timmer W.A., Bruining A., "Determination of The Local Flow Angle on Rotating Blades", Proceedings of the World Wind Energy Conference and Exhibition, July-2002, Berlin, Germany.

${ }^{25}$ Gerber B.S, Tangler J.L, Duque E.P.N and Kocurek J.D, "Peak and Post Power Aerodynamics from Phase VI NASA Ames Wind Turbine Data", Proceedings of the Special Topic Conference: the Science of Making Torque from Wind, EWEA, Delft, The Netherlands, 2004.

${ }^{26}$ Tangler J.L., "Insight into Wind Turbine Stall and Post-stall Aerodynamics", Wind Energy, Vol 7, 2004, pp247-260.

${ }^{27}$ Giguere P., Selig M.S., "Design of a Tapered and Twisted Blade for the NREL combined Experiment Rotor", NREL/SR500-26173, NREL, Golden, CO, 1999.

${ }^{28}$ Hand M.M, Simms, Fingersh L.J., Jager D.W., Cortell J.R., Schreck S. and Larwood, S.M., "Unsteady Aerodynamics Experiments Phase VI: Wind Tunnel Test Configurations and Available Data Campaigns", NREL Technical Report NREL/TP500-29955, Golden, Colorado, Dec 2001.

${ }^{29}$ Leishman J.G., Bhagwat, M.J., and Bagai, A, "Free-Vortex Filament Methods for the Analysis of Helicopter Rotor Wakes", Journal of Aircraft, Vol. 39, No. 5, pp 759-775, 2002.

${ }^{30}$ Bhagwat, M.J. and Leishman, J.G., "Generalized Viscous Vortex Model for Application to Free-Wake and Aeroacoustic Calculations," $58^{\text {th }}$ Annual Forum and Technology Display of the American Helicopter Soc. Intern., Montreal, Canada, 2002.

${ }^{31}$ Ananthan, S, Leishman J.G., Ramasamy, M, "The Role of Filament Stretching In The Free-Vortex Modelling of Rotor Wakes, $58^{\text {th }}$ Annual Forum and Technology Display of the American Helicopter Society International, Montreal, Canada, 2002. 